\title{
HARDWARE IMPLEMENTATION OF A TAKAGI-SUGENO NEURO-FUZZY SYSTEM OPTIMIZED BY A POPULATION ALGORITHM
}

\author{
Piotr Dziwiński ${ }^{1, *}$, Andrzej Przybył ${ }^{1}$, \\ Paweł Trippner ${ }^{2,4}$, Józef Paszkowski ${ }^{3,4}$, Yoichi Hayashi ${ }^{5}$ \\ ${ }^{1}$ Department of Computer Engineering, Czestochowa University of Technology, \\ al. Armii Krajowej 36, 42-200 Czestochowa, Poland \\ ${ }^{2}$ Management Department \\ University of Social Sciences, 90-113 Łódź \\ ${ }^{3}$ Information Technology Institute \\ University of Social Sciences, 90-113 Łódź \\ ${ }^{4}$ Clark University Worcester, MA 01610, USA \\ ${ }^{5}$ Meiji University, Tama-ku, Kawasaki, 214-8571 Japan \\ ${ }^{*}$ E-mail: piotr.dziwinski@pcz.pl
}

Submitted: 7th September 2020; Accepted: 19th April 2021

\begin{abstract}
Over the last several decades, neuro-fuzzy systems (NFS) have been widely analyzed and described in the literature because of their many advantages. They can model the uncertainty characteristic of human reasoning and the possibility of a universal approximation. These properties allow, for example, for the implementation of nonlinear control and modeling systems of better quality than would be possible with the use of classical methods. However, according to the authors, the number of NFS applications deployed so far is not large enough. This is because the implementation of NFS on typical digital platforms, such as, for example, microcontrollers, has not led to sufficiently high performance. On the other hand, the world literature describes many cases of NFS hardware implementation in programmable gate arrays (FPGAs) offering sufficiently high performance. Unfortunately, the complexity and cost of such systems were so high that the solutions were not very successful. This paper proposes a method of the hardware implementation of MRBF-TS systems. Such systems are created by modifying a subclass of Takagi-Sugeno (TS) fuzzy-neural structures, i.e. the NFS group functionally equivalent to networks with radial basis functions (RBF). The structure of the MRBF-TS is designed to be well suited to the implementation on an FPGA. Thanks to this, it is possible to obtain both very high computing efficiency and high accuracy with relatively low consumption of hardware resources. This paper describes both, the method of implementing MRBFTS type structures on the FPGA and the method of designing such structures based on the population algorithm. The described solution allows for the implementation of control or modeling systems, the implementation of which was impossible so far due to technical or economic reasons.
\end{abstract}

Keywords: hardware implementation of fuzzy systems, FPGA, population algorithms 


\section{Introduction}

The typical structure of a Takagi-Sugeno Neuro-Fuzzy System (TS-NFS) is as follows

$$
\operatorname{IF}\left(\mathbf{x} \text { is } \overline{\mathbf{x}}^{j}\right) \operatorname{THEN}\left(y^{j}=a_{0}^{j}+\mathbf{a}^{j} \cdot \mathbf{x}\right),
$$

where

$$
\mathbf{x}=\left[x_{1}, \cdots, x_{N}\right]^{T},
$$

$\mathbf{x}$ is a vector with a size of $N$ representing the input to the system. The $\overline{\mathbf{x}}^{j}$ defines an input fuzzy set for the $j$-th fuzzy rule $(j=1 . . M)$. While, the $a_{0}^{j}$ and

$$
\mathbf{a}^{j}=\left[a_{1}^{j}, \quad \cdots \quad, a_{N}^{j}\right]
$$

defines an output value for the $j$-th rule in the Takagi-Sugeno system.

In the considered systems Gaussian input fuzzy sets are used. The algebraic product is used as a T-norm operator. Rules consequents are a singleton type and the method of the center of gravity for singletons (COGS) is used for defuzzification. For the sake of clarity, a system with one exit $(y)$ will be considered in this paper. However, it should be noted that such a limitation does not lead to the loss of generality of the presented method. According to what was presented in the publication $[1,2]$, a system with such properties can be treated as functionally equivalent to a system with radial basis functions. The sets described by Hyper Radial Basis Functions (HRBF) are used herein. Such a system equipped with a Takagi-Sugeno type output was presented in the publication [3] as MRBF-TS, ie a modified radial basis system with Takagi-Sugeno output.

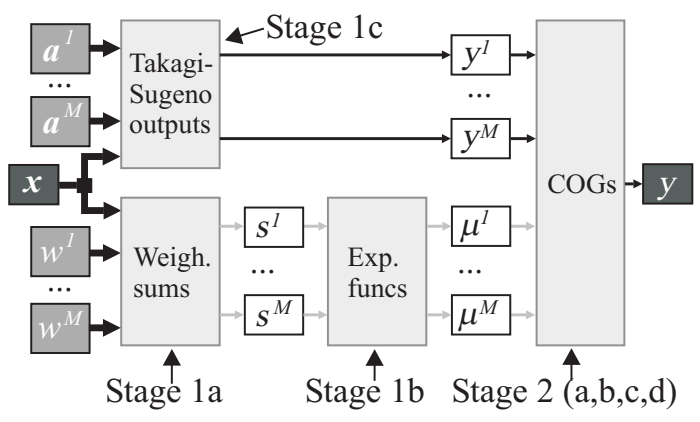

Figure 1. The first stage of calculations.

In the analyzed class of systems, the process of determining the $(y)$ output value can be performed in two stages (Figure 1, Figure 2). In the first stage, the values of the membership functions of the system input for all $M$ of multidimensional fuzzy sets of the HRBF type are determined (1a, 1b). Also, the output values of these rules are determined based on the Takagi-Sugeno method (1c). In the second stage, based on the determined degrees of activation of HRBF sets, the output values of all rules are aggregated based on the COGS method. In particular, it should be noted that, the stage 1a of the described algorithm, according to the MRBF-TS method, comes down to determining the distance of the input vector from the centers of the HRBF sets. Therefore, the following calculations should be made for each of the $M$-rules of the analyzed system:

$$
\begin{aligned}
s^{j}=w_{0}^{j}+ & w_{1,1}^{j} x_{1}+w_{1,2}^{j}\left(x_{1}\right)^{2}+\ldots \\
& +w_{N, 1}^{j} x_{N}+w_{N, 2}^{j}\left(x_{N}\right)^{2} .
\end{aligned}
$$

In the above formula, there are numerical constants defining the shape and distribution of HRBF sets, determined as follows:

$$
\begin{aligned}
& w_{0}^{j}=\left(\frac{\bar{x}_{1}^{j}}{\sigma_{1}^{j}}\right)^{2}+\ldots+\left(\frac{\bar{x}_{N}^{j}}{\sigma_{N}^{j}}\right)^{2}, \\
& w_{i, 1}^{j}=-\frac{2 \bar{x}_{i}^{j}}{\left(\sigma_{i}^{j}\right)^{2}}, w_{i, 2}^{j}=\left(\frac{1}{\sigma_{i}^{j}}\right)^{2} .
\end{aligned}
$$

The values $\bar{x}_{i}^{j}$ and $\sigma_{i}^{j}$ defines centers and widths of HRBF for $i$-th input $(i=1 . . N)$ of $j$-th rule. As it can be seen, the calculations presented by the formula (4) are based on repetitive operations such as multiplication and addition, which (in particular when using fixed-point arithmetic) can be efficiently performed in FPGA technology [3, 4].

The stage $1 \mathrm{~b}$ is the determination of the value of the membership function as follows:

$$
\mu^{j}=\exp \left(-\left\|\mathbf{x}-\overline{\mathbf{x}}^{j}\right\|^{2}\right)=\exp \left(s^{j}\right)
$$

The exponential function in the formula (7) is quite troublesome to implement on an FPGA. However, this function is only evaluated once for each rule. The value of this function can be determined with high precision using floating-point arithmetic $[5,6,7]$, fixed-point arithmetic [8], or the value can be estimated with limited precision using a look-up table (LUT) method [4].

The use of the LUT-based method for a function representing the degree of activation of rules is 
justified in many practical cases. It causes a significant reduction in the computation time. However, it requires a significant amount of RAM. The examples of the hardware implementation parameters of an exponential function are shown in Table 4. This issue will be analyzed later in this paper.

The stage $1 \mathrm{c}$ may be performed simultaneously with the stages $1 \mathrm{a}$ and $1 \mathrm{~b}$. In this stage, $y^{j}$ outputs of all fuzzy rules are determined as shown in their successors in the formula (1).

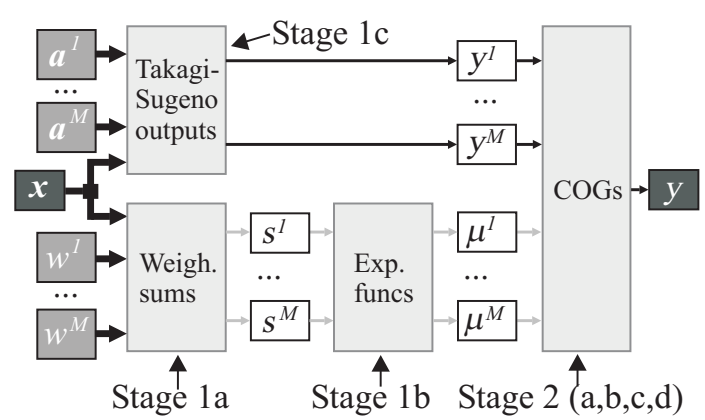

Figure 2. The second stage of the calculation according to the COGS method.

As previously announced and shown in Figure 2 , the second stage uses the values of the activation function $\mu^{j}$ calculated in the first stage and the output values of $y^{j}$ of all fuzzy rules to determine the output values of the entire system. For this purpose, the outputs of all system rules are aggregated according to the COGS method and Figure 2. In particular, in stages 2a-c, values $n$ and $r$ are determined for the multiplication operation (8) required in the final stage of the COGs defuzzification process, i.e. in the stage $2 \mathrm{~d}$.

$$
y=n \cdot r .
$$

To determine $r$, it is required to perform the sum of the activation function (stage 2a) of all rules (9) and then to calculate the arithmetic reciprocal (stage $2 b$ ) of this quantity (10), i.e. respectively:

$$
d=\sum_{j=1}^{M} \mu^{j}
$$

and

$$
r=\frac{1}{d}
$$

In turn, the determining of the value of $n$ requires performing a weighted sum (stage 2c) represented by the formula (11).

$$
n=\sum_{j=1}^{M} \mu^{j} \cdot y^{j}
$$

From the hardware implementation point of view, it is important that the stages $2 a-b$ and $2 c$ can be performed at the same time by the independent execution units.

Similarly to the first stage, also in this case the necessary calculations were mostly reduced to simple operations such as multiplication and addition. Moreover, it is necessary to implement one complex function, namely the arithmetic reciprocal of real numbers (10). The reciprocal operation can be performed either by a numerical algorithm or by an LUT-based method. Although the latter uses a significant amount of RAM, it is nevertheless justified in some applications.

Summing up, it can be stated that the implementation of the NFS structure can be reduced to the necessity of the performance of a fairly large number of elementary operations such as multiplication and addition. Moreover, it is necessary to perform a small number of complex operations determining the value of nonlinear functions of one variable, i.e. exp and reciprocal.

\begin{tabular}{ll} 
QUALITY & PARAMETERS OF IMPLEMENTED \\
INDICATORS & PROCESSING SYSTEM \\
\hline REACTION TIME & PROCESSING RESOLUTION \\
\hline ACCURACY & PUMBER OF FUZZY RULES \\
\hline COST
\end{tabular}

Figure 3. The idea of multi-criteria optimization in the hardware implementation of NFS.

Based on the above analysis, it can be concluded that when proceeding to the next stages of the hardware implementation of NFS in the FPGA technology, certain decisions must be made that have a very large impact on the obtained results. Well, as shown in Figure 3, the obtained solution can be evaluated based on the following quality indicators: reaction time, accuracy and cost (manufacturing and operating).

The reaction time determines the speed of the system, i.e. the time after which the system will 
process the input data and calculate the appropriate response.

The accuracy of the system determines the precision with which the system performs the tasks entrusted to it, e.g. approximation, modeling, identification or control. This indicator may be determined by the root-mean square error (RMSE) value or the maximum value of the absolute error.

The cost of manufacturing is related to a very diverse price of FPGAs with different amounts of hardware resources (see the last column in the Table 1). On the other hand, the cost of operation results mainly from the amount of electricity consumed. In some types of applications, it is very important to minimize electricity consumption. First of all, mobile systems powered by batteries or from renewable energy sources should be mentioned here.

The reduction of electricity consumption also reduces heat losses and allows for the implementation of a working system without the need for a fan to cool such a system. In many practical systems, harsh environmental conditions make it necessary to operate without a fan. Therefore, the aspect of minimizing electricity consumption is very important also in the case of designing stationary systems, powered from the mains.

The value of quantified quality indicators is primarily influenced by three parameters of the implemented system, namely: processing resolution, number of fuzzy rules and the degree of parallelization of calculations. This effect is symbolically indicated in Figure 3.

Processing resolution is primarily the number of bits used to represent real signals processed in a digital system. It is obvious that - to a certain extent - the higher the processing resolution, the higher the precision of the entire system is possible [9]. However, this comes at the cost of the increased complexity of the digital circuit necessary to be implemented in FPGA technology. As a result, the production cost and electricity consumption increase, and the response time of such a solution increases (the speed of operation decreases).

Apart from the width of the binary word, the applied standard of binary coding of real numbers also has a significant impact on the obtained precision. Namely, it is possible to use floating-point or fixed-point arithmetic. Both cases will be analyzed later in this work.

To confirm the importance of this issue, the results of research contained in various publications can be cited. For example, [10] describes "dynamic execution profiling tool designed to identify where an application can benefit from reduced precision or reduced range in floating-point computations." In the conclusions, the authors sum up "results show that applications often have highly modal value distributions, offering promise for aggressive floatingpoint arithmetic optimizations." They also wrote that "in applications where a very narrow range is required, fixed point arithmetic may be appropriate. Using pure integer arithmetic provides a dramatic performance improvement or decrease in cost when compared to floating-point".

The authors of [11] also note that "across a set of applications spanning the domains of DSP, robotics, and machine learning ... hot loops in the applications can be perforated by an average of $50 \%$ with a proportional reduction in execution time, while still producing an acceptable quality of results. In addition, the width of the data used in the computation can be reduced to $10-16$ bits from the currently common 32/64 bits with potential for significant performance and energy benefits."

In turn, in the publication [12], the authors propose the "Float-Fix" format, which, as they show, is an efficient and hardware-friendly data type for neural networks. They also explain that the loss of precision due to using such a format is less than $0.12 \%$. However, its use allows for a significant reduction in the cost of hardware implementation of the algorithm of deep neural networks.

In this paper we will show that in many practical cases it is possible to obtain a satisfactory precision with a reduced width of the processed digital words, i.e. to obtain an optimal solution. This means that the requirements of a specific application will be met with the implementation costs reduced to a minimum and minimum electricity consumption. As part of the analysis carried out in the next section, it will be shown that increasing the number of fuzzy rules while reducing the processing resolution can lead to an economical optimization of the system. 


\section{Analysis of numerical problems}

Consider the following MRBF-TS system example with one input and one output. Let us assume that in this system we have two rules of the HRBF type. The parameters of radial sets are as follows: $\bar{x}_{1}^{1}=0.0, \sigma_{1}^{1}=0.3, \bar{x}_{1}^{2}=1.0$ and $\sigma_{1}^{2}=0.3$. Whereas, the parameters of the successors of the two applied rules are: $a_{o}^{1}=0.0, a_{1}^{1}=0.0, a_{0}^{2}=1.0$ and $a_{1}^{2}=0.0$.
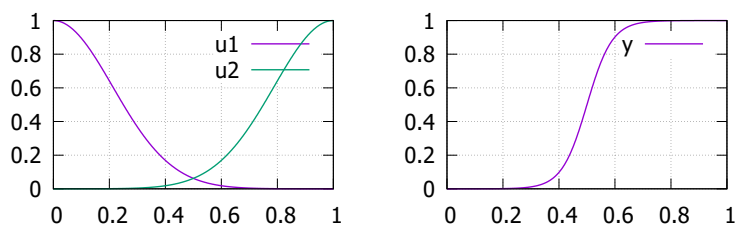

Figure 4. Examples of signal values expected in a digital implementation of a fuzzy system.

Figure 4 shows the forms of the obtained membership functions and the value generated at the output of such a system $(y)$ respectively from the left side. The hardware implementation of such a system described further, uses a fairly simple and efficient 8-bit fixed-point arithmetic in FPGA. In this case, analogous waveforms shown in Figure 5 are obtained, where the symbols with an asterisk represent the values reproduced by the digital system.
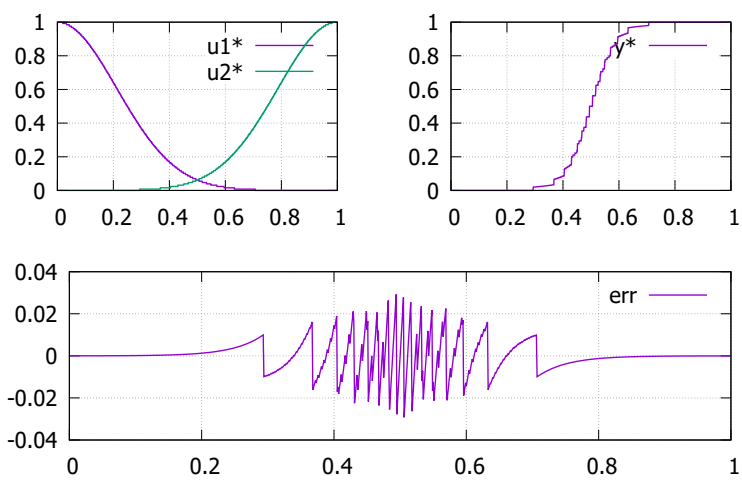

Figure 5. Signals generated by an 8-bit digital unit.

At the bottom of Figure 5 it is shown the error $\left(e r r=y-y^{*}\right)$ resulting from the implementation of a fuzzy system on a digital platform with a limited resolution of the processed digital words. As it can be seen, the maximum value of the measurement error is approximately $3 \%$ of the maximum value. Let us assume, for example, that the obtained result is not satisfactory because the designer expects an error of no more than $1 \%$ of the maximum value. It is possible to reduce the error value in at least two ways, i.e. by increasing the binary word processing resolution in the digital system or increasing the number of radial sets used to describe such a system.

It is obvious that if the processing resolution is increased (by lengthening the binary words) then the quality of the waveform $\left(y^{*}\right)$ reproduced by the digital system will be improved. For example, the experiment carried out in the analyzed case shows that the absolute value of the error dropped to a value below $0.7 \%$ (i.e. to the required level) when the resolution of the processed digital words was increased to 10-bits.
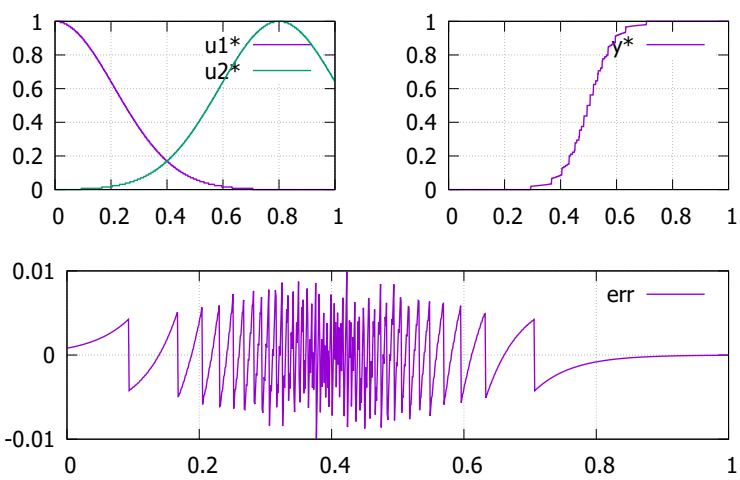

Figure 6. Signals generated by an 8-bit digital unit.

As shown in the next experiment (Figure 6), a similar effect can be obtained if the distances between adjacent fuzzy sets are slightly reduced $\left(\bar{x}_{1}^{2}=\right.$ 0.8 ). In this case, the required effect is achieved without the need to increase the width of the binary words processed.

The analysis of both cases described here (shown in Figure 5 and Figure 6) leads to the conclusion that the numerical problem shown here is most visible when the output values of the neighboring rules are significantly different, while their sets inputs only slightly overlap. In particular, it can be seen in Figure 5, where the value runs of $\mu_{1}^{*}$ and $\mu_{2}^{*}$ intersect at the low values, close to the resolution of the processed digital words. Reducing the distance between adjacent sets or increasing their width (Figure 6) avoids this phenomenon.

As already shown in Figure 6, the quality of the approximation has improved and the maximum value of the approximation error has decreased to the acceptable level. The important point here is that this was achieved without having to increase the processing resolution in the hardware- 
implemented digital system. However, it was necessary to select slightly different parameters of the fuzzy sets. This therefore results in the need to increase their number, in order to obtain a sufficiently precise description of the entire NFS.

The presented analysis, therefore shows that it is possible to achieve the assumed goal (expressed here with the acceptable value of the approximation error) by using: (a) high processing precision and an appropriate number of fuzzy rules or (b) using lower processing precision but at the same time increasing the number and modifying the parameters of such sets accordingly.

Thus, an important conclusion is that it is necessary to take into account the parameters of the hardware implementation at the stage of designing its structure to obtain an optimized solution.

\section{Hardware implementation of arithmetic operations in FPGA}

It should be noted that both previously presented approaches, i.e. increasing the processing precision and the number of fuzzy rules, lead to an increase in computational complexity and thus increase the cost of the hardware implementation. Which approach will be better in a particular application depends on many factors and should be subject to a compromise taking into account not only performance and precision but also the cost of the solution being developed.

These factors are influenced, among others, by parameters of the hardware implementation system such as the level of parallelism and the resolution of digital signal processing used.

The concept of the degree of parallelization of computations implies the information on the extent to which the implemented algorithm's computations are performed in a parallel manner. This term should be understood as the execution of various fragments of the processed algorithm simultaneously by the independent execution units. Parallel execution of calculations allows for a significant increase in processing efficiency compared to serial execution. Unfortunately, this happens at the expense of a significant demand for hardware resources, which increases the cost of such an implementation.
It is optimal in many cases if only a certain part of the computation is performed in parallel and the rest of the computation is performed in series.

Taking the right decisions in the scope of the above-mentioned issues will allow us to obtain the expected result at the lowest possible cost. However, it is not an easy task. In practice, in most cases, it is done by trial and error although this method is time-consuming. A much better way is to use a multi-criteria optimization algorithm that will automate this process. In further sections of this publication, the multi-criteria optimization algorithm will be designed and tested to support the process of designing the hardware implementation of the analyzed class of neural-fuzzy systems.

It should be noted that the general task of decomposing any algorithm intended for hardware implementation into elementary execution blocks is not a simple issue and exceeds the scope of this publication. However, in the case of the implementation of the analyzed NFS class, this task is significantly simplified. This is due to the fact that as shown earlier - most of the calculations required for execution, i.e. (4), (11) and (9) are based on repetitive of multiply and accumulate (MAC) operations. These types of calculations are quite simple and relatively easy to implement in FPGA technology. MAC operations are commonly used in digital signal processing (DSP) applications. Examples are: matrix and vector multiplication operations (commonly used in modeling the dynamics of various types of objects), convolution operations (used for example in the implementation of convolutional networks), and the implementation of various types of control algorithms.

Many publications present various approaches to the hardware implementation of the weighted sum described by the formulas (4), (11) and (9). Among others, the work [3] presents calculations describing the number of required work cycles necessary to perform the above-mentioned operations in a serial or parallel manner for an exemplary implementation. In the cited work it was assumed that a single work cycle corresponds to the execution time of an elementary arithmetic operation.

In this paper, much more reliable calculations will be presented in relation to this issue. Namely, the possibility of working of arithmetic units with different widths of the binary word and based on 
different coding standards for real numbers will be taken into account. Various structures for seriesparallel processing will also be analyzed. For this purpose, the properties of currently available, modern FPGAs, i.e. the 7-series Xilinx, were analyzed. Xilinx is one of the few leading manufacturers of this type of integrated circuits on the market. Selected resources of representative systems are included in the Table 1.

Table 1. Basic parameters of selected Xilinx "7 series" FPGAs.

\begin{tabular}{lllll}
\hline $\begin{array}{l}\text { FPGA } \\
\text { part number }\end{array}$ & $\begin{array}{l}\text { DSP } \\
\text { slices }\end{array}$ & $\begin{array}{l}\text { CLB } \\
\text { slices }\end{array}$ & BRAMs & $\begin{array}{l}\text { Approx. } \\
\text { price }[\$]\end{array}$ \\
\hline \hline XC7A12T & 40 & 2000 & 40 & 35 \\
\hline XC7A50T & 120 & 8,150 & 150 & 100 \\
\hline XC7A200T & 740 & 33,650 & 730 & 350 \\
\hline XC7K480T & 1,920 & 74,650 & 1,910 & $5 \mathrm{k}$ \\
\hline XC7VX980T & 3,600 & 153,000 & 3,000 & $18 \mathrm{k}$ \\
\hline
\end{tabular}

The first column of the table lists the symbolic names of the selected digital circuits, in order from the layouts with the smallest to the biggest resources. The most important resource (from the point of view of the hardware implementation of MRBF_TS systems) is "DSP Slices". Their number in specific systems is shown in the second column of the table. They are hardware blocks that perform elementary arithmetic operations (i.e. addition and multiplication) on integers. These blocks are also used to assist with certain operations with floating-point numbers. It should be noted that a single arithmetic operation may require from one to several such blocks. How many DSP Slices will be used to perform the arithmetic operation depends mainly on the width of the binary word being processed. Examples of the demand for DSP slices by various types of arithmetic operations are presented in the Tables 2, 3, 4 and 5.

In practical cases, it may turn out that the designed hardware processing algorithm requires more DSP slices than offered by the selected FPGA chip. In such a situation it is possible to use universal programmable logic instead of DSP slices, grouped in CLB slices (third column of the Table 1). However, in this case, in some applications, the speed of the arithmetic operation performed in this way is reduced. Each of FPGA CLB slice contains four LUTs (i.e. 6-input look-up tables) and eight registers.

In the Tables 2, 3, 4 and 5, arithmetic operations based solely on CLB slices are marked with an "L" at the end. For example, the symbol FX16L is a fixed-point arithmetic operation performed without the use of DSP slices, while the symbol FX16 is an analogous arithmetic operation performed with their use. Similarly, the symbols FL32 and FL16 denote floating-point arithmetic, 32-bit, and 16-bit, respectively.

Fixed-point arithmetic is widely used in the field of digital signal processing in FPGA $[13,4]$ technology. Its significant advantage is that it is built based on integer arithmetic, which is easy to implement on FPGA. The disadvantage, in turn, is that appropriate scaling of the real numbers encoded in this way is required at each processing stage. Although this is not a complicated arithmetic operation, the necessity to use it causes that the design of the processing system becomes noticeably more complicated. Despite this, fixed-point operations are unrivaled in systems that require the highest processing performance [4].

In some cases, it is also possible to use the LUT-based method to approximate the value of a function of one variable. In this case, the values of the approximated functions are calculated offline and placed in the cells of the table. For this purpose, dedicated hardware blocks containing RAM (BRAM) can be used. FPGAs usually contain from several dozen to several thousand such blocks, as shown in the fourth column of the Table 1 . With some simplification it can be said that each memory block has a capacity of $16 \mathrm{~Kb}$ and can be organized as 8,16 or 32 bit memory.

The last column of the table shows the approximate market price of a single FPGA chip. There are significant differences in price between systems with different amounts of resources. In the case of designing systems in which the economic aspect is important (it is rather the majority of practical systems), this parameter should also be taken into account. 
Table 2. The obtained parameters of the hardware implementation of the arithmetic multiplication operation.

\begin{tabular}{lllll}
\hline $\begin{array}{l}\text { Coding } \\
\text { standard }\end{array}$ & Latency DSP & LUTs & Regs \\
slices & & \\
\hline \hline FL32 & 3 & 2 & 138 & 180 \\
\hline FL16 & 2 & 1 & 70 & 77 \\
\hline FX32 & 3 & 4 & 71 & 163 \\
\hline FX24 & 1 & 2 & 49 & 114 \\
\hline FX24L & 3 & 0 & 647 & 555 \\
\hline FX18 & 1 & 1 & 29 & 73 \\
\hline FX18L & 2 & 0 & 372 & 183 \\
\hline FX16 & 1 & 1 & 21 & 65 \\
\hline FX16L & 2 & 0 & 300 & 177 \\
\hline FX14 & 1 & 1 & 29 & 57 \\
\hline FX14L & 2 & 0 & 234 & 155 \\
\hline FX12 & 1 & 1 & 21 & 49 \\
\hline FX12L & 2 & 0 & 172 & 121 \\
\hline FX10 & 1 & 1 & 21 & 41 \\
\hline FX10L & 2 & 0 & 122 & 101 \\
\hline FX8 & 1 & 1 & 17 & 33 \\
\hline FX8L & 1 & 0 & 81 & 49 \\
\hline & & & &
\end{tabular}

FPGAs with a large number of resources are also characterized by a high consumption of electricity. Power consumption is roughly proportional to the amount of hardware resources used. In this article, under the broadly understood concept of "cost" of the hardware implementation, we understand jointly: the cost associated with the market purchase price, the amount of electricity consumption and the resulting possible need to use cooling fans, as well as the complexity and dimensions of the designed system. The cost defined in this way will be expressed (with some simplification) by the amount of consumed hardware resources, which translates indirectly to the above-mentioned factors.

In the optimization algorithm proposed in the next section, the preference will be given to solutions that perform the task entrusted to them at the lowest possible cost. As it will be shown later, this will be achieved largely by using hardware processing based on arithmetic working with the smallest binary word widths possible.
Table 3. The obtained parameters of the hardware implementation of the arithmetic addition operation.

\begin{tabular}{lllll}
\hline $\begin{array}{l}\text { Coding } \\
\text { standard }\end{array}$ & Latency & $\begin{array}{l}\text { DSP } \\
\text { slices }\end{array}$ & LUTs & Regs \\
\hline \hline FL32 & 5 & 2 & 228 & 224 \\
\hline FL16 & 3 & 0 & 195 & 133 \\
\hline FX32 & 1 & 1 & 45 & 97 \\
\hline FX32L & 1 & 0 & 77 & 129 \\
\hline FX24 & 1 & 1 & 29 & 73 \\
\hline FX24L & 1 & 0 & 53 & 97 \\
\hline FX18 & 1 & 1 & 20 & 55 \\
\hline FX18L & 1 & 0 & 28 & 73 \\
\hline FX16 & 1 & 1 & 21 & 49 \\
\hline FX16L & 1 & 0 & 37 & 65 \\
\hline FX14 & 1 & 1 & 18 & 43 \\
\hline FX14L & 1 & 0 & 32 & 57 \\
\hline FX12 & 1 & 1 & 11 & 37 \\
\hline FX12L & 1 & 0 & 23 & 49 \\
\hline FX10 & 1 & 1 & 8 & 31 \\
\hline FX10L & 1 & 0 & 18 & 41 \\
\hline FX8 & 1 & 1 & 5 & 25 \\
\hline FX8L & 1 & 0 & 13 & 33 \\
\hline
\end{tabular}

In this study, experimental research was conducted to determine, among other things how many clock cycles (for an exemplary, but representative implementation) are required by individual arithmetic operations. Detailed data on the operations of multiplication, addition, determination of the value of the exponential function and arithmetic reciprocal are summarized in the following Tables 2, 3, 4, and 5 respectively.

The values presented in these tables were obtained as a result of an experimental study carried out for an exemplary but representative XC7A200T FPGA chip (Table 1). Based on this data, it was possible to compare the reaction time of a hardware implemented fuzzy system, the cost of its implementation, and the obtained precision.

As intended earlier, the hardware implementation can be realized based on the processing of signals of different binary word widths and based on different binary real number coding standards.

In particular, the Table 2 shows the most important performance and cost metrics for an arithmetic multiplication hardware implementation. Slightly similar results were also presented in other publica- 
tions, for example in the work [13]. It shows that in the case of the implementation of fixed-point multipliers, doubling the width of the binary word (for example - from the FX16 to FX32 format) results in an approximately fourfold increase in the demand for hardware resources and a twofold reduction in the maximum possible operating frequency. These results concerned the design of data processing systems in a single clock cycle.

Table 4. Analyzed parameters of the hardware implementation of the function exp.

\begin{tabular}{lllll}
\hline $\begin{array}{l}\text { Coding } \\
\text { standard }\end{array}$ & $\begin{array}{l}\text { Latency DSP } \\
\text { slices }\end{array}$ & $\begin{array}{l}\text { LUTs/ } \\
\text { Regs }\end{array}$ & BRAMs \\
\hline \hline FL32 [6] & 17 & 4 & $\begin{array}{l}1154 / \\
1154\end{array}$ & 1 \\
& & & $0 / 0$ & 16 \\
\hline FX14 & 2 & 0 & $0 / 0$ & 4 \\
\hline FX12 & 2 & 0 & $0 / 0$ & 1 \\
\hline FX10 & 2 & 0 & $0 / 0$ & 1 \\
\hline FX8 & 2 & 0 & $0 / 0$ & 1 \\
\hline
\end{tabular}

Table 5. Obtained parameters of the hardware implementation of the reciprocal function.

\begin{tabular}{lllll}
\hline $\begin{array}{l}\text { Coding } \\
\text { standard }\end{array}$ & Latency LUTs & Regs & BRAMs \\
\hline \hline FL32 & 28 & 819 & 1414 & 0 \\
\hline FL16 & 8 & 239 & 226 & 0 \\
\hline FX16L & 23 & 190 & 279 & 0 \\
\hline FX14L & 21 & 143 & 204 & 0 \\
\hline FX12L & 19 & 122 & 186 & 0 \\
\hline FX10L & 17 & 100 & 160 & 0 \\
\hline FX14 & 2 & 0 & 0 & 16 \\
\hline FX12 & 2 & 0 & 0 & 4 \\
\hline FX10 & 2 & 0 & 0 & 1 \\
\hline
\end{tabular}

It should be noted that different arithmetic operations may be performed with a different number of internal processing cycles. This parameter is expressed as an integer $p$ and is referred to as latency $(p)$. The use of high $p$ values (i.e. from a few to several dozens) allows to obtain, in the implementation of a specific arithmetic operation, high values of the maximum operating frequency of the digital system. However, the result of the operation carried out in this way is obtained only after $p+1$ clock cycles.

When designing a system composed of many cooperating arithmetic units, it should be assumed that each of them should work with a value of $p$ greater than zero. This approach enables better integration of the entire hardware data processing system. Moreover, in the case of data stream processing, for $p>0$ it is possible to obtain additional advantages of performing subsequent computations in a so-called pipe. Details concerning this issue, however, are beyond the scope of this work and will not be presented.

To take into account the practical aspects of implementation, it is necessary to establish a certain common operating frequency of all arithmetic units used. In the experiment, the frequency $f=200 \mathrm{MHz}$ was selected and on this basis the required value of $p$ was determined for each operation. The results in the Tables 2, 3, 4, and 5 apply to this case.

As shown in the previous section, a similar end result of the hardware implementation of the MRBF-TS system can be obtained by using different real number encoding standards. Whereby, operations processing binary data with larger binary word width or using floating-point arithmetic require more hardware resources and a longer time to process the input data (expressed by a larger value of the $p$ parameter). However, they offer higher processing precision.

\section{Architecture of hardware imple- mentation of MRBF-TS systems}

In this section, various possibilities of configuring the hardware computing systems in MRBF-TS systems will be presented.

The extensive possibilities of designing the hardware processing architecture offer a great opportunity to optimize this process. Unfortunately, this also makes it quite a complex process. Therefore, the solution presented below does not exhaust this topic. They should rather be treated as an open problem regarding the optimization of such systems.

As mentioned before, any kind of binary arithmetic can be used at the various stages of the hardware processing of MRBF-TS systems (Figure 1). As a consequence, a system with different parameters (quality-cost) can be created. As a result of the analysis of numerical problems, it turned out that not all (theoretically possible) configurations of various types of arithmetic are justified. For this 
reason, a selection of a number of different binary processing standards configurations considered in this paper, appropriate for each stage of the hardware implementation of MRBF-TS systems, was selected.

The Table 6 contains the analyzed configurations for computing the fuzzy set membership function. In turn, the Table 7 contains the analyzed configurations for the inference and fuzzyfication process.

Table 6. Analyzed configurations of binary coding standards at individual stages of determining the value of the membership function of HRBF sets.

\begin{tabular}{lll}
\hline $\begin{array}{l}\text { Stage (Eq.) } \\
\backslash \text { ICIndex }\end{array}$ & $\begin{array}{l}\text { 1a } \\
(4)\end{array}$ & $\begin{array}{l}\text { lb } \\
(7)\end{array}$ \\
\hline \hline 1 & FL32 & FL32 \\
\hline 2 & FL16 & FX14 \\
\hline 3 & FL16 & FX12 \\
\hline 4 & FX24 & FX14 \\
\hline 5 & FX18 & FX14 \\
\hline 6 & FX16 & FX14 \\
\hline 7 & FX14 & FX14 \\
\hline 8 & FX14 & FX12 \\
\hline 9 & FX12 & FX12 \\
\hline 10 & FX12 & FX10 \\
\hline 11 & FX10 & FX10 \\
\hline 12 & FX10 & FX8 \\
\hline 13 & FX8 & FX8 \\
\hline
\end{tabular}

Table 7. Analyzed configurations of binary coding standards at the stages of inference and defuzzyfication of the MRBF-TS system.

\begin{tabular}{lllll}
\hline $\begin{array}{l}\text { Stage (Eq.) } \\
\backslash \text { OCIndex }\end{array}$ & $\begin{array}{l}\text { 1c } \\
(1)\end{array}$ & $\begin{array}{l}\text { 2a,2c } \\
(11,9)\end{array}$ & $\begin{array}{l}\text { 2b } \\
(10)\end{array}$ & $\begin{array}{l}\text { 2d } \\
(8)\end{array}$ \\
\hline \hline 1 & FL32 & FL32 & FL32 & FL32 \\
\hline 2 & FL16 & FL16 & FL16 & FL16 \\
\hline 3 & FX24 & FX24 & FX16L & FX24 \\
\hline 4 & FX18 & FX18 & FX16L & FX18 \\
\hline 5 & FX16 & FX16 & FX16L & FX16 \\
\hline 6 & FX16 & FX16 & FX14 & FX16 \\
\hline 7 & FX14 & FX14 & FX16L & FX14 \\
\hline 8 & FX14 & FX14 & FX14 & FX14 \\
\hline 9 & FX12 & FX12 & FX14 & FX12 \\
\hline 10 & FX10 & FX10 & FX12 & FX10 \\
\hline 11 & FX8 & FX8 & FX10 & FX8 \\
\hline
\end{tabular}

In the further part of the work, the considered configurations will be identified by two integers, i.e. ICIndex $=1 \ldots 13$ as described in the Table 6 and OCIndex $=1 \ldots 11$ as described in the Table 7 .

As mentioned before, and shown in Figure1, stages $1 \mathrm{a}$ and $1 \mathrm{~b}$ must be performed sequentially. However, stage $1 \mathrm{c}$ is performed in parallel with them. Similarly, stages $2 a(9)$ and $2 b$ (10) must be performed sequentially. In contrast, stage $2 c$ (11) can be processed at the same time. Upon completion, the stage $2 \mathrm{~d}$, represented by the equation (8), begins.

It follows that the response time (or the latency time) of the entire hardware-implemented MRBFTS system (expressed in the number of necessary $c_{s}$ calculation cycles) is determined as follows:

$$
\begin{aligned}
c_{s} & =\max \left(c_{1 \mathrm{a}}+c_{1 \mathrm{~b}}, c_{1 \mathrm{c}}\right) \\
& +\max \left(c_{2 \mathrm{a}}+c_{2 \mathrm{~b}}, c_{2 \mathrm{c}}\right)+c_{2 \mathrm{~d}},
\end{aligned}
$$

where the symbols $c_{1 a} \cdots 2 d$ represent the delay times (expressed in the number of computational cycles) for the stages identified by subscripts, as indicated in the Figure 1.

Consequently, the response time of the designed system $\left(T_{s}\right)$ can be estimated in seconds. This value results from the designated reaction time multiplied by the clock cycle time, ie $T_{s}=c_{s} \cdot T_{f}$, where $T_{f}=\frac{1}{f}$.

However, it should be realized that - due to the complexity of the system - this is only an estimation. The numerical values indicated here should be confirmed each time through experimental tests carried out as part of a specific application. In the described method, it is more important to compare different hardware implementation methods with each other than to precisely analyze the obtained numerical values.

The method for determining the delay times and the amount of resources used for individual stages of the hardware implementation of the MRBF-TS system in its various configurations will be presented below. The method of determining the amount of required hardware resources will also be presented.

One of the most important elements of the described hardware computing architecture is the way of organizing the computations in stages $1 \mathrm{a}$ and $1 \mathrm{~b}$. In particular, this remark applies to systems with a large number of inputs and/or a large number of 
fuzzy rules. This is because the individual rules can at this stage be processed in parallel (Figure 8), serial (Figure 7) or serial-parallel. As mentioned before, the choice of the method of computing organization carries serious consequences both in terms of the demand for hardware computing units and the obtained processing speed.

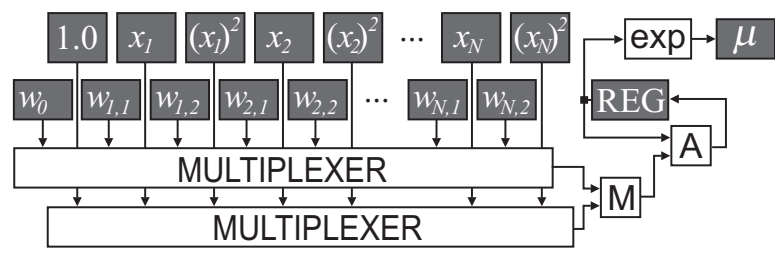

Figure 7. General idea of serial data processing described by the equation (4).

For the computation in serial form (Figure 7), the value of $c_{r}$ denoting the delay expressed by the number of clock cycles necessary to determine the degree of activation of a single HRBF set (7) is determined as follows:

$$
c_{r}^{s e r}=2 N \cdot \max \left(c_{M 1}, c_{A 1}\right)+c_{A 1}+c_{e},
$$

where: $c_{\mathrm{M} 1}$ i $c_{\mathrm{A} 1}$ are the number of clock cycles (i.e. $1+p$ ) for the multiplication and addition operations, respectively, according to the data contained in the Tables 2 and 3 for the arithmetic used in stage $1 \mathrm{a}$.

In turn, the value of $c_{e}=1+p$ is an analogous value related to the data contained in the Table 4. The value of $c_{e}$ is independent of whether the above-mentioned we perform calculations in a parallel or serial manner.

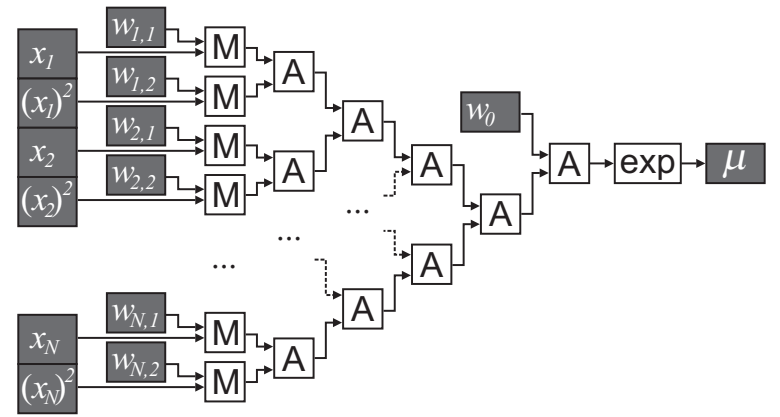

Figure 8. General idea of parallel data processing described by the equation (4).

The approach described by the equation (13) offers the lowest performance among the various hardware calculation methods of the equation (4) representing the activation value of a single HRBF rule. However, this serial mechanism has the ad- vantage of a very low hardware resource requirement (i.e. arithmetic processing units). In this case, only one multiplier and one adder are required, ie $m_{r}^{\text {ser }}=1$ and $a_{r}^{\text {ser }}=1$, respectively.

In the parallel solution (Figure 8) we expect the highest possible performance of computations in hardware, accepting quite a significant demand for FPGA hardware resources. In this case, it is possible to perform all the multiplication operations contained in the equation (4) simultaneously, using $m_{r}^{p a r}=2 N$ arithmetic multipliers working in parallel. However, the partial results are summed in pairs in the following ceil $\left(\log _{2} 2 N\right)$ cycles [3]. For this, it is necessary to allocate $a_{r}^{\text {pairs }}=2 N$ adders. As a result of the above-mentioned analysis, you can determine the necessary number of cycles for the hardware implementation of the equation (7) in a parallel way:

$$
c_{r}^{p a r}=c_{M 1}+\operatorname{ceil}\left(\log _{2} 2 N\right) \cdot c_{A 1}+c_{A 1}+c_{E},
$$

"where, "ceil( )" is rounded up to an integer."

Based on the above analysis, the following formula can be determined describing the number of computational cycles required to determine the degree of activation of a single HRBF rule:

$$
c_{r}=c_{r}^{\text {par }} \cdot P_{R}+c_{r}^{\text {ser }} \cdot\left(1-P_{R}\right),
$$

where $P_{R}=\{0,1\}$ - is a parameter that defines whether the calculation of the degree of activation of a single rule (7) is performed in parallel $\left(P_{R}=1\right)$ or in series $\left(P_{R}=0\right)$.

The demand for hardware resources can be expressed analogously. In particular, the number of adders required is:

$$
a_{r}=a_{r}^{\text {par }} \cdot P_{R}+a_{r}^{\text {ser }} \cdot\left(1-P_{R}\right),
$$

while, the number of required arithmetic multiplication units is:

$$
m_{r}=m_{r}^{\text {par }} \cdot P_{R}+m_{r}^{\text {ser }} \cdot\left(1-P_{R}\right) .
$$

It should also be noted that the abovementioned calculations relate to the procedure for determining the degree of activation of a single HRBF rule. In a typical case, we are dealing with many rules (usually from a few to a dozen). Therefore, it should also be considered whether individual rules should be processed sequentially or in parallel. In the first case, the time of necessary calculations is proportionally extended by $M$, while the 
demand for FPGA hardware resources does not increase significantly. In the second case, the calculation time remains constant, while the resource consumption increases $M$ - fold, i.e. in proportion to the number of processed rules. Intermediate solutions are also possible, combining the advantages of both of the above solutions.

As you can easily check the number of cycles required to determine the value of the activation function of all the rules is:

$$
c_{\mu}=c_{r} \cdot \operatorname{ceil}\left(\frac{M}{P_{S}}\right),
$$

where the parameter $P_{S}=1,2, . ., M$ defines how numerous is the group of $\mathrm{HRBF}$ rules processed in parallel, i.e. by independent execution units working at the same time. In case when $\left(P_{S}<M\right)$, calculations for successive groups are performed sequentially, which is included in the formula (18).

It should be noted that, in accordance with the markings shown in Figure 1, $c_{1 a}+c_{1 b}=c_{\mu}$.

Similarly, the hardware resource requirements for stages $1 \mathrm{a}$ and $1 \mathrm{~b}$ are: $a_{1 a}=a_{r} \cdot P_{S}, m_{1 a}=m_{r} \cdot P_{S}$ and $e_{1 b}=P_{S}$.

As it is easy to check, analogous parameters can be determined for stage $1 \mathrm{c}$ as follows:

$$
c_{1 c}=c_{y} \cdot \operatorname{ceil}\left(\frac{M}{P_{S}}\right)
$$

where $c_{y}$ expresses the number of cycles required to determine the output value of a single rule, based on the Takagi-Sugeno formula, i.e. the right side of the equation 1. This value can be determined as follows:

$$
c_{y}=c_{y}^{p a r} \cdot P_{R}+c_{y}^{s e r} \cdot\left(1-P_{R}\right)
$$

where

$$
c_{y}^{\text {ser }}=N \cdot \max \left(c_{M 1}, c_{A 1}\right)+c_{A 1},
$$

and

$$
c_{y}^{p a r}=c_{M 1}+\operatorname{ceil}\left(\log _{2} 2 N\right) \cdot c_{A 1}+c_{A 1} .
$$

In turn, the required number of adding and multiplying arithmetic units is, respectively:

$$
a_{y}=a_{y}^{p a r} \cdot P_{R}+a_{y}^{s e r} \cdot\left(1-P_{R}\right)
$$

$$
m_{y}=m_{y}^{p a r} \cdot P_{R}+m_{y}^{s e r} \cdot\left(1-P_{R}\right),
$$

where: $a_{y}^{\text {ser }}=1, m_{y}^{\text {ser }}=1, a_{y}^{\text {par }}=N$ and $m_{y}^{\text {par }}=N$. Thus, the total number of arithmetic processing units required to implement stage $1 \mathrm{c}$ in hardware is: $a_{1 c}=a_{y} \cdot P_{S}$ and $m_{1 c}=m_{y} \cdot P_{S}$.

By analogy with the methods shown in Figures 7 and 8 , it can easily be shown that the processing time of stage $2 \mathrm{a}$ (expressed as the number of clock cycles) is:

$$
c_{2 a}=c_{2 a}^{p a r} \cdot P_{Y}+c_{2 a}^{s e r} \cdot\left(1-P_{Y}\right),
$$

where $P_{Y}=\{0,1\}$ - is a parameter that defines whether the calculations regarding the defuzification of the system output value are performed in parallel $\left(P_{Y}=1\right)$ or in series $\left(P_{Y}=0\right)$. On the other hand, the symbols $c_{2 a}^{\text {ser }}$ and $c_{2 a}^{p a r}$, similarly to the previous cases, define the hardware processing time of the equation (9), respectively in a serial manner (Figure 7) or parallel (Figure 8). These values are determined as follows:

$$
c_{2 a}^{\text {ser }}=1+M \cdot c_{A 2},
$$

and

$$
c_{2 a}^{p a r}=c_{M 2}+\operatorname{ceil}\left(\log _{2} M\right) \cdot c_{A 2}+c_{A 2} .
$$

The symbols $c_{A 2}, c_{M 2}$ denote the number of clock cycles for the addition and multiplication operation, respectively, in the 2 nd stage. These values are determined from the Tables 3 and 2 in a manner analogous to determining the values of $c_{A 1}, c_{M 1}$. In this case the OCIndex is used as the configuration identifier for the real-number binary coding standard used. The $c_{2 b}$ and $c_{2 d}$ parameters for stages $2 \mathrm{~b}$ and $2 \mathrm{~d}$ are read from the Tables 5 and 2, respectively.

The parameters of the hardware implementation of stage $2 \mathrm{c}$, by analogy to stages $1 \mathrm{a}$ and $2 \mathrm{a}$, can be described as follows:

$$
c_{2 c}=c_{2 c}^{\text {par }} \cdot P_{D}+c_{2 c}^{\text {ser }} \cdot\left(1-P_{D}\right),
$$

where $P_{D}=\{0,1\}$ - is a parameter that defines whether the calculations for the defuzzification of the system output value are performed in parallel $\left(P_{D}=1\right)$ or in series.

On the other hand, the symbols $c_{2 c}^{\text {ser }}$ and $c_{2 c}^{\text {par }}$, similarly to the previous cases, define the hardware processing time of the equation (11), respectively in 
a serial way (Figure 7) or parallel (Figure 8). These values are determined as follows:

$$
c_{2 c}^{s e r}=M \cdot \max \left(c_{A 2}, c_{M 2}\right)+c_{A 2},
$$

and

$$
c_{2 c}^{p a r}=c_{M 2}+\operatorname{ceil}\left(\log _{2} M\right) \cdot c_{A 2}+c_{A 2} .
$$

Similarly, it can also be shown that the following number of arithmetic addition and multiplication units is necessary for the implementation of stage 2a:

$$
\begin{gathered}
a_{2 a}=a_{2 a}^{p a r} \cdot P_{D}+a_{2 a}^{s e r} \cdot\left(1-P_{D}\right), \\
m_{2 a}=m_{2 a}^{p a r} \cdot P_{D}+m_{2 a}^{s e r} \cdot\left(1-P_{D}\right),
\end{gathered}
$$

where: $a_{2 a}^{\text {ser }}=1, m_{2 a}^{\text {ser }}=0, a_{2 a}^{\text {par }}=M-1$ and $m_{2 a}^{\text {par }}=$ 0 .

For the stage $2 \mathrm{c}$, analogous values are determined as follows:

$$
\begin{aligned}
& a_{2 c}=a_{2 c}^{p a r} \cdot P_{D}+a_{2 c}^{s e r} \cdot\left(1-P_{D}\right), \\
& m_{2 c}=m_{2 c}^{p a r} \cdot P_{D}+m_{2 c}^{s e r} \cdot\left(1-P_{D}\right),
\end{aligned}
$$

where: $a_{2 c}^{\text {ser }}=1, m_{2 c}^{\text {ser }}=1, a_{2 c}^{\text {par }}=M-1$ and $m_{2 c}^{\text {par }}=$ $M$.

In turn, stage $2 b$ requires a single "reciprocal" operation i.e.

$$
r_{2 b}=1,
$$

while, stage $2 \mathrm{~d}$ a single multiplication operation, i.e.

$$
m_{2 d}=1 \text {. }
$$

For the entire system, the number of arithmetic operations (multiplication, addition, "exp" and "reciprocal", respectively), performed by independent hardware resources is:

$$
\begin{gathered}
m_{s}=m_{1 a}+m_{1 c}+m_{2 a}+m_{2 c}+m_{2 d} . \\
a_{s}=a_{1 a}+a_{1 c}+a_{2 a}+a_{2 c}, \\
e_{s}=e_{1 b}, \\
r_{s}=r_{2 b},
\end{gathered}
$$

The demand for hardware resources of FPGAs, expressed in the number of DSP slices, LUTs, registers and BRAMs, is determined based on the Tables 2, 3, 4 and 5 .
The number of DSP units used is calculated by the following formula:

$$
\begin{aligned}
\operatorname{Num}^{D S P} & =D_{S P}+D S P_{a} \\
& +\operatorname{Num}_{e}^{D S P}(1 b, \text { ICIndex }) \\
& +\operatorname{Num}_{r}^{D S P}(2 b, \text { OCIndex })
\end{aligned}
$$

where: $D S P_{m}, D S P_{a}$ - the number of DSP units used to perform multiplication and accumulation operations at all stages of the hardware implementation of the system, $N_{u m} m_{e}^{D S P}$ - is a function returning the number of DSP units used at stage $1 b$ to perform the exp operation (Table 4) according to the selected ICIndex (Table 6), Num ${ }_{r}^{D S P}$ - is a function returning the number of DSP units used at stage $2 b$ according to the selected OCIndex (Table 5).

The number of DSP units required to perform the multiplication operation for the entire system is given by the formula

$$
\begin{aligned}
D S P_{m} & =\operatorname{Num}_{m}^{D S P}\left(m_{1 a}, \text { ICIndex }, C L B_{1 a-m}\right)(42) \\
& +\operatorname{Num}_{m}^{D S P}\left(m_{1 c}, \text { OCIndex }, C L B_{1 c-m}\right) \\
& +\operatorname{Num}_{m}^{D S P}\left(m_{2 a}, \text { OCIndex }, C L B_{2 a 2 c-m}\right) \\
& +\operatorname{Num}_{m}^{D S P}\left(m_{2 c}, \text { OCIndex }, C L B_{2 a 2 c-m}\right) \\
& +\operatorname{Num}_{m}^{D S P}\left(m_{2 d}, \text { OCIndex }, C L B_{2 d-m}\right)
\end{aligned}
$$

where: $\operatorname{Num}_{m}^{D S P}\left(m_{e}, \operatorname{Index}, C L B\right)$ - is a function that determines the number of DSP units required to perform the multiplication operation at a given stage of the computation $e, e \in\{1 a, 1 c, 2 a, 2 c, 2 d\}$ according to the Table 2 determined from the value of the Index depending on the choice of the version of the multiplication operator used $C L B_{p-m}, p$ - is the computation stage, $p \in\{1 a, 1 c, 2 a 2 c, 2 d\}$, according to the choice of the version of the multiplication operator encoded in the solution vector $x^{M R B F-T S}$ - the formula (62) (for example: version FX16 or FX16L).

On the other hand, the required number of DSP units needed for the accumulation operation can be determined by the formula

$$
\begin{aligned}
D S P_{a} & =N U M_{a}^{D S P}\left(a_{1 a}, \text { ICIndex }, C L B_{1 a-a}\right)(43) \\
& +N U M_{a}^{D S P}\left(a_{1 c}, \text { OCIndex }, C L B_{1 c-a}\right) \\
& +N U M_{a}^{D S P}\left(a_{2 a}, \text { OCIndex }, C L B_{2 a 2 c-a}\right) \\
& +N U M_{a}^{D S P}\left(a_{2 c}, \text { OCIndex }, C L B_{2 a 2 c-m}\right)
\end{aligned}
$$

where: $N U M_{a}^{D S P}\left(a_{e}\right.$, Index,$\left.C L B\right)$ - is a function that determines the number of DSP units required to perform accumulation at a given stage of the computation $e, e \in\{1 a, 1 c, 2 a, 2 c\}$ according to the Table 3 
determined by the value of the Index depending on the choice of the version of the multiplication operator used $C L B_{p-a}, p$ - is the computation stage, $p \in\{1 a, 1 c, 2 a 2 c\}$, according to the choice of the version of the accumulation operator encoded in the solution vector $x^{M R B F-T S}$ - formula (62).

Similarly, the number of Regs and LUTs required can be determined by replacing the DSP function with one that returns the number of occupied Regs and LUTs from the appropriate tables for the specified OCIndex and UCIndex indices and versions of the arithmetic operators used $C L B_{p-q}$

The number of BRAMs used is determined by the formula

$$
N_{u m}^{B R A M s}=\operatorname{ceil}\left(\frac{N u m^{B}}{2000}\right)
$$

where:

$$
\begin{aligned}
\text { Num }^{B} & =B_{1 a}+B_{1 b}+B_{1 c} \\
& +B_{2 a}+B_{2 b}+B_{2 c}+B_{2 d}
\end{aligned}
$$

where: $B_{1 a}$ - is the number of bytes used in stage 1a of the calculation to write the weights $\left[w_{0}^{1}, \ldots, w_{0}^{M}\right],\left[w_{1,1}^{1}, \ldots, w_{N, 1}^{M}\right],\left[w_{1,2}^{1}, \ldots, w_{N, 2}^{M}\right]$, system inputs $\left[x_{1}, \ldots, x_{N}\right],\left[\left(x_{1}\right)^{2}, \ldots,\left(x_{N}\right)^{2}\right]$ and the results of summing $\left[s^{1}, \ldots, s^{M}\right]$, that is in total:

$$
B_{1 a}=N_{u m}^{B}(\text { ICIndex }) \cdot 2(N+M+N \cdot M)
$$

$B_{1 b}$ - is the number of bytes used in stage $1 \mathrm{~b}$ of the calculations to save the resulting values of the membership function $\left[\mu^{1}, \ldots, \mu^{M}\right]$ and optionally in case of using the exponent $N U M_{\text {exp }}^{B}$ (ICIndex), that is in total:

$$
\begin{aligned}
B_{1 b} & =N_{1 b}^{B}(\text { ICIndex }) \cdot M \\
& +N_{\text {umm }}^{B}(\text { ICIndex })
\end{aligned}
$$

$B_{1 c}$ - is the number of bytes used in stage $1 \mathrm{c}$ of the calculations to save the parameters of the function for the Takagi-Sugeno part $\left[a_{0}^{1}, \ldots, a_{0}^{M}\right],\left[a_{1}^{1}, \ldots, a_{N}^{M}\right]$ and the calculated result values $\left[y^{1}, \ldots, y^{M}\right]$, that is, in total

$$
B_{1 c}=N_{u m}^{B}(\text { OCIndex }) \cdot(2 M+M \cdot N)
$$

$B_{2 a}$ - requires only a certain number of bytes to write one sum result, i.e. $B_{2 a}=N u m_{2 a}^{B}, B_{2 b}$ - is the number of bytes used to save the results division and, optionally, an array in the case of reciprocal $N_{u m}^{B}$ rec $($ OCIndex $)$ arrays, i.e.

$$
B_{2 b}=N_{2 b}^{B}+N_{\text {rec }}^{B} \text { (OCIndex) }
$$

At stage $2 \mathrm{c}$ and $2 \mathrm{~d}$, only the received results need to be stored in the implemented system, so the number of bytes for these stages is $B_{2 c}=N u m_{2 c}^{B}$ (OCIndex) and $B_{2 d}=N_{u m}^{B}$ (OCIndex). The calculation of the required number of BRAMs blocks does not take into account the area needed to convert the data domain at the input of the system as well as at its output.

At this point, it should be recalled that the above-mentioned calculations include only the most important, from the point of view of the analyzed application, resources of FPGA circuits, i.e. those required to implement arithmetic units. Other resources, which are also necessary to perform other functions of the system, have been omitted. However, the number of these omitted resources is significantly lower and will not be analyzed as it does not affect the essence of the presented algorithm. The purpose of the described method is not to indicate the optimal solution in the strict sense of the word. Rather, it is about obtaining a nearly optimal solution in an automated manner.

\section{Description of the multi-criteria optimization approach}

This Section describes the quality evaluation algorithm of the obtained the hardware implementation of the MRBF-TS system on the FPGA platform, taking into account all the early mentioned evaluation criteria.

The value of the numerically defined quality indicators is mainly influenced by three parameters of the implemented system, namely: the resolution of processing, the number of fuzzy rules, and the degree of computational parallelization. This influence has been symbolically marked in Figure 3 .

The most crucial element of a multi-criteria optimization algorithm is the quality evaluation function of the obtained solution. Its proper construction enables the algorithm to follow a solution that is a compromise between particular evaluation criteria under defined constraints. 
As already mentioned, three basic indicators will be used to evaluate the quality of the obtained solution: the performance (reaction time), the accuracy, and the cost of the solution.

The obtained accuracy of the hardware implementation consists of two factors - the root mean square error RMSE and the maximum value from the absolute value of the errors $\max \left(\left|y-y^{*}\right|\right)$. The cost of the obtained solution consists of the number of DSP units, Regs, LUTs used, and the number of BRAMs blocks used.

All the evaluation criteria mentioned earlier take values from different ranges. For this reason, to balance the influence of the used criteria on the solution quality evaluation function, it is necessary to normalize the values of these components to a consistent range of values. In the described algorithm, it is proposed to use the normalization of the evaluation criteria by using the maximum values of these criteria considered acceptable in the obtained solution by the expert. The normalization is implemented using the formula:

$$
\tilde{e c_{k}}=\frac{e c_{k}}{e c_{k}^{\max }}
$$

where: $e c_{k}-$ is the value of the given evaluation criterion, $k=1, \ldots, 7$ - the number of the used criterion according to the Table. $8, e c_{k}^{\max }$ - is the maximum value allowed in the obtained solution. In this way, all evaluation criteria were reduced to the uniform interval $[0,1]$. If $\left(\tilde{e c_{k}}>1\right)$ is obtained, it is known that the criterion is not satisfied and the impact of this criterion on the evaluation of the quality of the solution should have a bigger impact.

In this paper, the quality of the hardware implementation of the HRBF-TS system is evaluated using the three evaluation criteria shown in Figure 3 . The system operation accuracy $E^{A}(\tilde{\mathbf{e c}})$ is determined using RMSE function as follows:

$$
E^{A}(\tilde{\mathbf{e c}})=\sqrt{\frac{\left(\tilde{e c_{1}}\right)^{2}+\left(\tilde{\left.e c_{2}\right)^{2}}\right.}{2}}
$$

The cost of the hardware implementation of the MRBF-TS system $E^{C}(\tilde{\mathbf{e c}})$ can be calculated using a similar formula

$$
E^{C}(\tilde{\mathbf{e c}})=\sqrt{\frac{\sum_{k=3}^{6}\left(\tilde{e} c_{k}\right)^{2}}{4}}
$$

On the other hand, the speed of the system (reaction time) is the value of the $\tilde{e c_{7}}$ parameter (Table 8).

Table 8. Limiting values (maximum allowable values) for individual criteria of the evaluation of the hardware implementation.

\begin{tabular}{lll}
\hline $\begin{array}{l}\text { Evaluation } \\
\text { criteria } e c_{k}\end{array}$ & $\begin{array}{l}\text { Criterion } \\
\text { index } \\
k\end{array}$ & $\begin{array}{l}\text { Max value } \\
e c_{k}^{\max }\end{array}$ \\
\hline \hline RMSE & 1 & $1 \%$ \\
\hline $\max \left(\left|y-y^{*}\right|\right)$ & 2 & $2 \%$ \\
\hline Num $^{D S P}$ & 3 & 50 \\
\hline Num $^{\text {Regs }}$ & 4 & 1000 \\
\hline Num $^{\text {LUTs }}$ & 5 & 1500 \\
\hline Num $^{\text {BRAMs }}$ & 6 & 40 \\
\hline Latecy $_{s}$ & 7 & 165 \\
\hline
\end{tabular}

The evaluation criterion described by the formula (52) works very well when the boundary requirements of the evaluated criteria $e c_{k}^{\max }$ are met, that is for the value of $\left(\tilde{e} c_{k} \leq 1\right)$. During the multiple processes of estimating the values of the evaluated criteria based on the exemplary shape of the function, it is possible to obtain the value of the cost criterion function $\left(E^{C}<1\right)$, despite not meeting (to a significant degree) the components of the limit criteria $e c_{k}^{\max }$. Such a situation is shown in Figure 12a, which was created as a result of composing the cost criteria components presented in Figure 10 and Figure 11 using the formula (52).

These figures show the $\tilde{e c_{k}}$ cost criteria values for all coding combinations described using OCIndex and ICIndex according to the Tables 6 and 7. These figures were created for the exemplary MRBF-TS system containing $(M=9)$ fuzzy rules, for the serial configuration of all operations, which means $P_{R}=0, P_{S}=0, P_{D}=0, P_{Y}=0$, and the selection of DSP slices to perform arithmetic operations. It should be noted that the target cost of the obtained implementation may be lower, which results directly from the number of fuzzy rules selected by the population algorithm. 
a) Normalized Max $\left|y-y^{*}\right|$

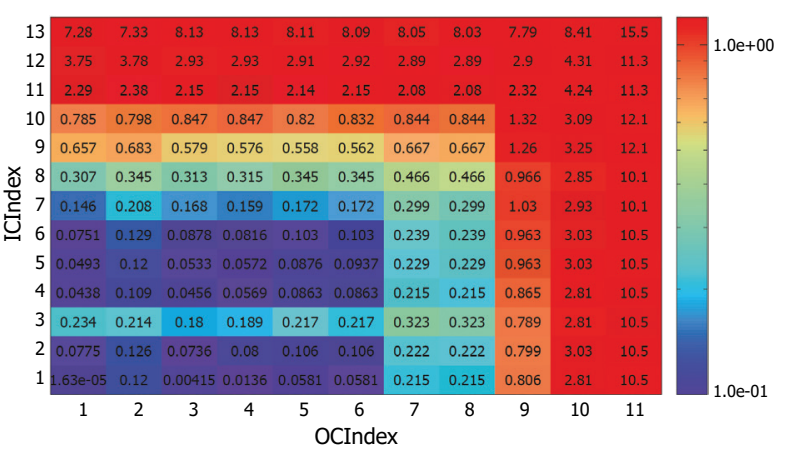

b) Normalized RMSE error

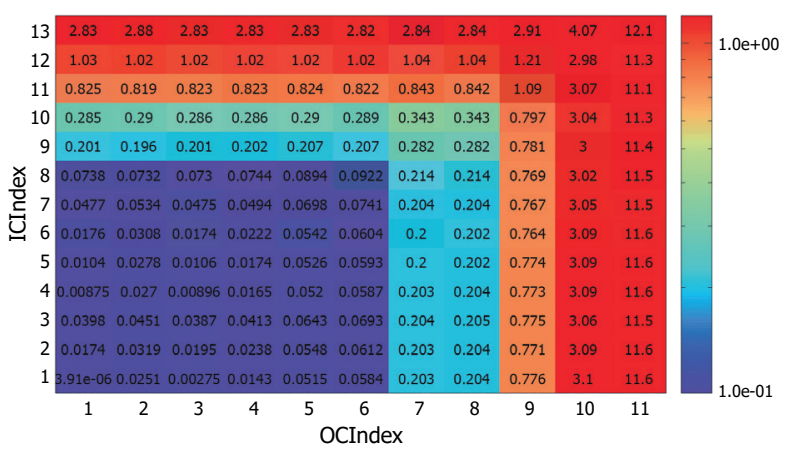

c) Precision of the system

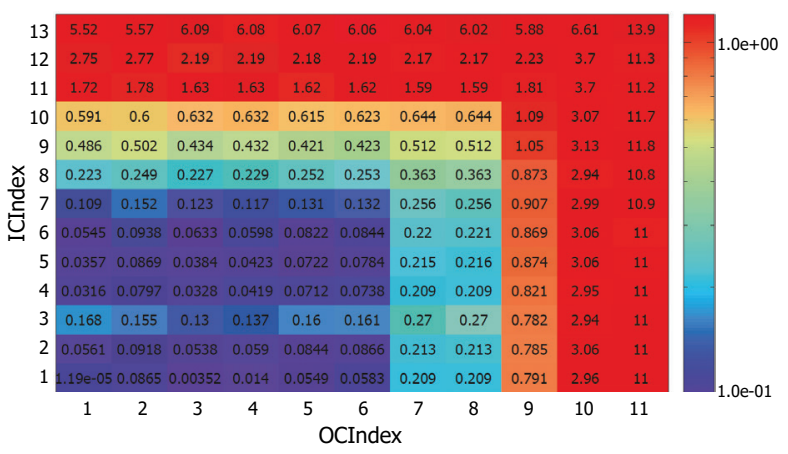

Figure 9. The obtained values of the evaluations criteria influencing to the evaluation of the accuracy of the hardware implementation of the MRBF-TS.

a) Degree of use the maximum Regs number

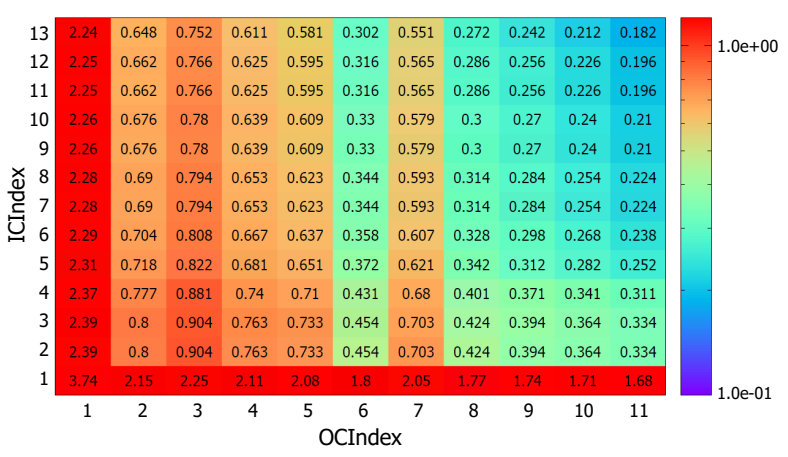

b) Degree of use the maximum LUTs number

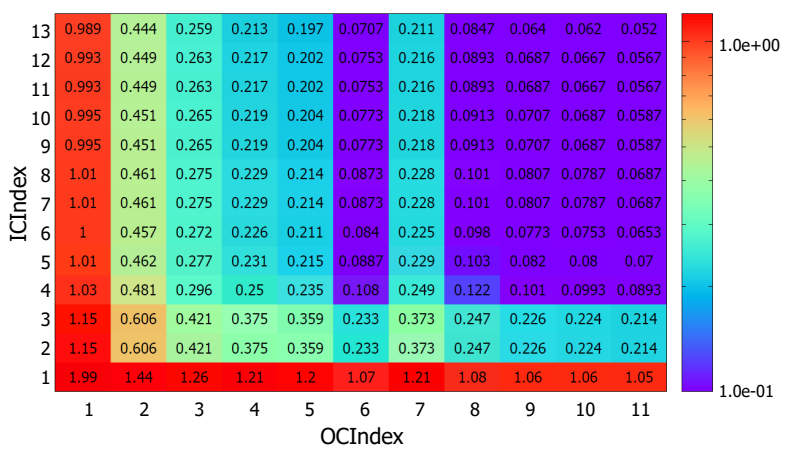

Figure 10. The obtained values of the evaluations criteria (Regs and LUTs) influence to the evaluation of the cost of the hardware implementation of the MRBF-TS.

a) Degree of use the maximum DSP slice number

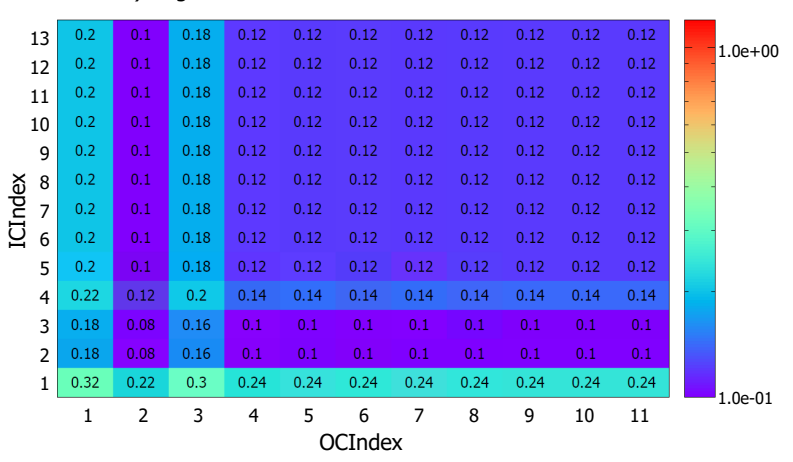

b) Degree of use the maximum BRAMs block number

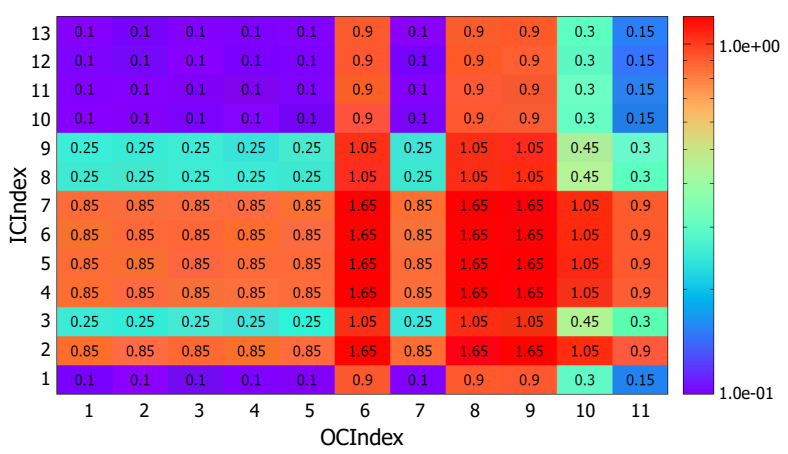

Figure 11. The obtained values of the evaluations criteria (DSP slices and BRAMs) influence to the evaluation of the cost of the hardware implementation of the MRBF-TS.

Due to the necessity to meet the components of the limit criteria $e c_{k}^{\max }$ (there are hard criteria - only, in this case, a working hardware implementation can be created), it is proposed to use the criterion function max for values exceeding the limit criteria. The modified cost criterion function takes the following form 
a) Cost of the system (RMSE)

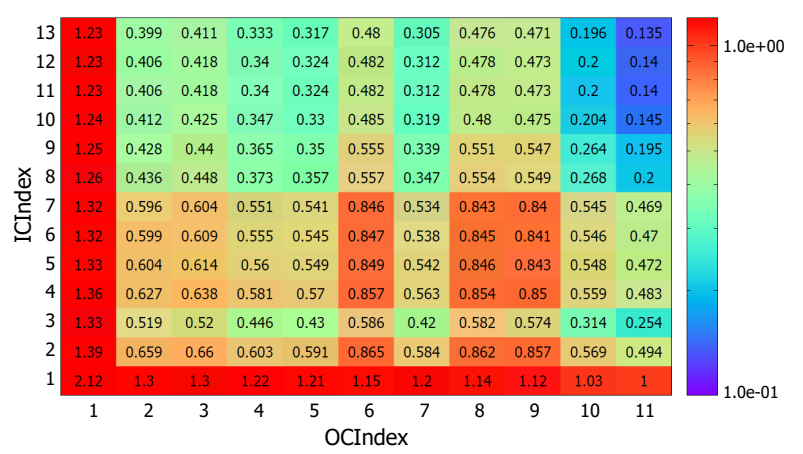

b) Cost of the system (MAX)

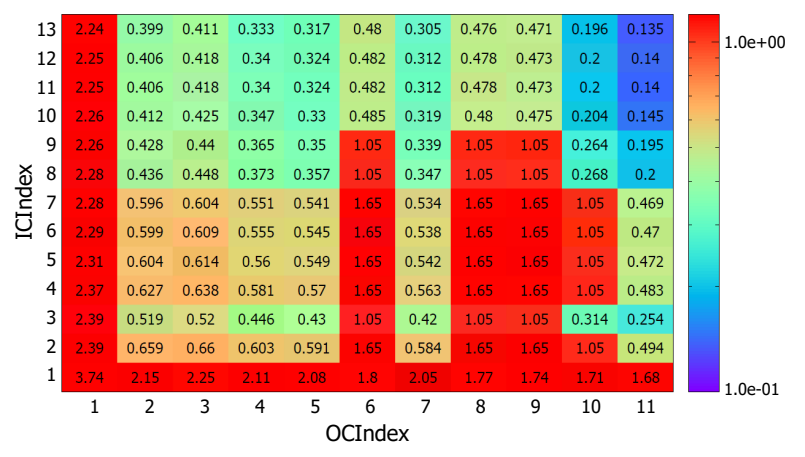

Figure 12. The obtained values of the evaluation of the cost of the hardware implementation of the HRBF-TS system: (a), (b) - using the formulas (52) and (53) respecivelly.

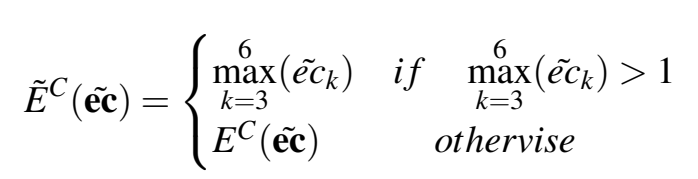

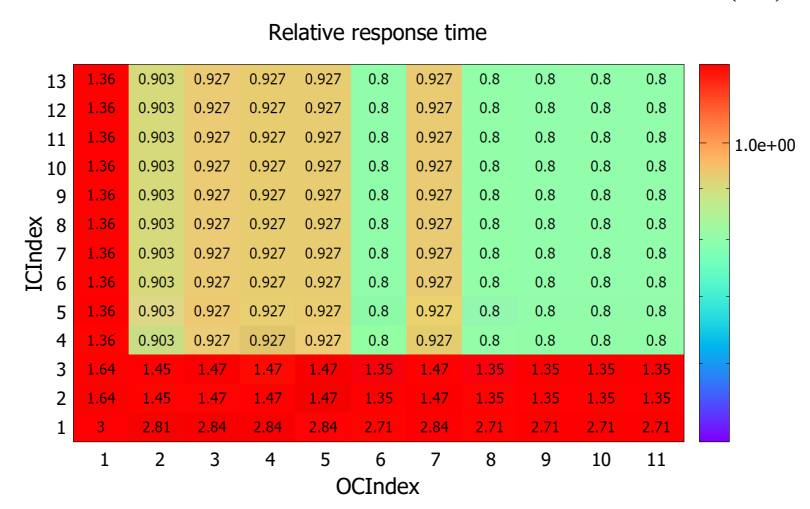

Figure 13. The obtained values of the performance evaluation of the hardware implementation of the HRBF-TS system.

This function forces the population algorithm to meet the criteria whose value is $\left(\tilde{e} c_{k}>1\right)$ in the first place. The result of using this cost function is shown in Figure 12b. A similar situation oc- curs in the case of aggregation of the main criteria: cost, accuracy, and performance (reaction time). If we apply the RMSE function according to the formulas (54) or (55), in the result we obtain evaluation $\left(E Q^{1}<1\right)$ or $\left(E Q^{2}<1\right)$, for cases where all evaluation criteria are not met. The described phenomenon can be observed in Figure 14a and Figure $14 \mathrm{~b}$.

$$
E Q^{1}=\sqrt{\frac{\left(E^{A}(\tilde{\mathbf{e c}})\right)^{2}+\left(E^{C}(\tilde{\mathbf{e c}})\right)^{2}+\left(\tilde{e} c_{7}\right)^{2}}{3}}
$$

or

$$
E Q^{2}=\sqrt{\frac{\left(E^{A}(\tilde{\mathbf{e c}})\right)^{2}+\left(\tilde{E}^{C}(\tilde{\mathbf{e c}})\right)^{2}+\left(\tilde{e c_{7}}\right)^{2}}{3}}
$$

Due to the presented drawback of using the RMSE measure - a modified version described by the formula 56 is proposed, similar to the cost function described by the formula 53 .

$$
\tilde{E Q}=\left\{\begin{array}{lll}
E Q^{\max } & \text { if } & E Q^{\max }>1 \\
E Q & & \text { othervise }
\end{array}\right.
$$

where

$$
E Q^{\max }=\max \left(E^{A}(\tilde{\mathbf{e c}}), \tilde{E}^{C}(\tilde{\mathbf{e c}}), \tilde{e c} c_{7}\right)
$$

The results of using the cost function (56) are shown in Figure 14c. Figure 14b illustrates the behavior of the solution quality function for evaluation using the formula (54), while Figure $14 \mathrm{c}$ shows the final results of the quality evaluation used by the population algorithm. This function guarantees that all required criteria for the quality evaluation of the solution are met (if it is possible).

\section{The Particle Swarm Optimiza- tion Algorithm}

a) Evaluation of the system (RMSE)

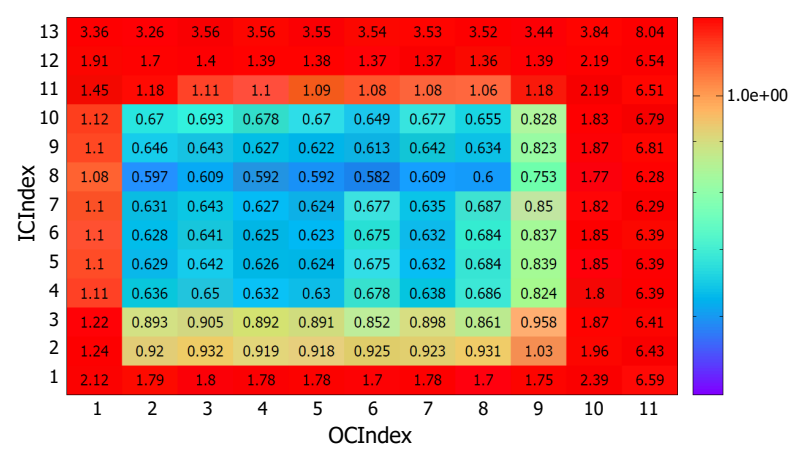




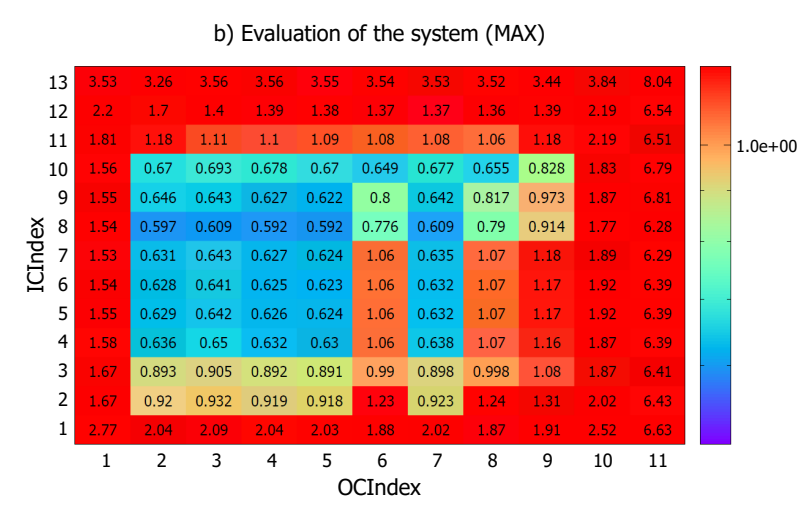

c) Evaluation of the system (MAX-MAX)

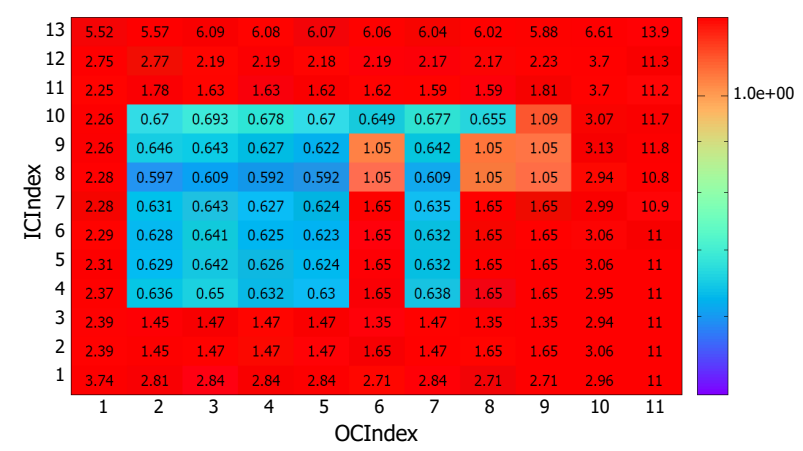

Figure 14. The obtained evaluation of the quality of the hardware implementation of the MRBF-TS system: (a) - using the formula (54), (b) - using the formula (55), (c) - using the formula (56).

The Particle Swarm Optimization Algorithm (PSO) was proposed by Eberhart and Russel in 1995 [14]. It is an iterative solution search method that goes through collections (swarms) of potential solutions $\mathbf{x}_{i}(t), i=1, \ldots, D, D$ the dimensional solution of the problem. Each potential solution is represented as a particle $a_{i}(t)=$ $\left(\mathbf{x}_{i}(t), \mathbf{v}_{i}(t), \mathbf{p}_{i}(t)\right)$, where $\mathbf{v}_{i}(t)$ is a non-zero velocity, $\mathbf{v}_{i}(t)=\left[v_{i}^{1}(t), \ldots, v_{i}^{D}(t)\right], \mathbf{p}_{i}(t)$ is the historical best solution of the $i$-th individual.

The position and velocity of particles are modified in each iteration of the algorithm according to the following formulas:

$$
\begin{aligned}
v_{i}^{d}(t+1) & =w \cdot v_{i}^{d}(t) \\
& +\psi_{1} \cdot r_{1} \cdot\left(p_{i}^{d}(t)-x_{i}^{d}(t)\right) \\
& +\psi_{2} \cdot r_{2} \cdot\left(g^{d}(t)-x_{i}^{d}(t)\right) \\
x_{i}^{d}(t+1) & =x_{i}^{d}(t)+v_{i}^{d}(t)
\end{aligned}
$$

where: $w$ - is the inertia coefficient, $w \in(0,1]$, $\psi_{1}$ and $\psi_{2}$ are constants controlling the effect of the local and global solution in the equation (58), $r_{1}$ i $r_{2}$ are uniform random numbers within the range $(0,1], \mathbf{g}(t)$ - is the best global solution for the entire population.

PSO algorithm for difficult optimization problems tends to converge prematurely manifested by a strong decrease in the values of the velocity of individuals and the increase in the homogeneity of the population. Mainly, for this reason, many modifications have been performed. Dziwiński et al. [15] proposed a hybrid method (FSHPSO-E) to combine the PSO algorithm with the Genetic Algorithm (GA) by replacing the solutions stored in the population with new better solutions discovered by the GA algorithm. They applied the control of the cooperation of algorithms using a fuzzy system of the MISO type trained on a set of benchmark functions. In the next work, [16] they introduce an auto adaptation mechanism that allows changing the form of fuzzy rules when solving the optimization problem. Łapa et al. [17] proposed a new population-based evolutionary algorithm that automatically configures the used search mechanism comes from various evolutionary algorithms.

In this paper, the FSHPSO-E-AA algorithm [16] is used for the task of designing and optimizing the hardware implementation of the MRBF-TS system for a selected approximation problem. It should be mentioned that the algorithm uses the knowledge acquired from optimizing a set of benchmark functions. The algorithm's task is focused on determining the parameters encoded according to the formula (62).

\section{Coding of the solution}

The hybrid population algorithm FSHPSO-EAA uses real encoding solutions. It is a consequence of the way population individuals move in a multidimensional solution space. Some of the parameters of the designed MRBF-TS system subject to hardware implementation are binary in nature $\{0,1\}$. These parameters are responsible for enabling/disabling specific configurations of the hardware implementation of the system. For all binary parameters, a function described by the formula (60) was used which converts a real value into a binary value. However, for integer values, rounding up was used according to the formula (61) 


$$
\begin{gathered}
\text { ceil_B }(q)= \begin{cases}1 & \text { if }(q \geq 0.5) \\
0 & \text { othervise }\end{cases} \\
\text { ceil_I }(q)=\operatorname{Round}(q+0.5)
\end{gathered}
$$

To facilitate the encoding of the solution in the real $\mathbf{x}^{M R B F-T S}$ format, a fixed-length vector is used that takes into account the maximum allowable number of rules in the system that can be used for a given problem. During the operation of the hybrid population algorithm, there is an automatic adaptation of the number of rules used by deactivating them $r a^{j}=0, j=1, \ldots, M$. When a rule is deactivated, the information about that rule contained in the solution $\mathbf{x}^{M R B F-T S}$ is not used.

Furthermore, at each stage of the hardware implementation of the MRBF-TS system, the algorithm selects the version of the arithmetic operators used - the version implemented by using the CLB slices $\left(C L B_{p-q}=1\right)$ or LUTs + Regs $\left(C L B_{p-q}=0\right)$, where $p$ is the calculation stage, $p \in\{1 a, 1 c, 2 a 2 c, 2 b, 2 d\}$ according to the Tables 6 and $7, q$ is the arithmetic operator used, $q \in\{a, m\}$, $a$ is the adder, $m$ is the multiplier. The choice of the versions of the arithmetic operators used at the computation stages directly affects the degree of use of hardware resources, as well as the performance (reaction time). The coded form of the solution in the form of a vector of real numbers was described by the formula (62).

$$
\mathbf{x}_{i}^{M R B F-T S}=\left\{\begin{array}{c}
r a^{1}, \ldots, r a^{M^{\max }} \\
\text { ICIndex, } O C I n d e x, \\
C L B_{1 a-a}, C L B_{1 a-m}, \\
C L B_{1 c-a}, C L B_{1 c-m}, \\
C L B_{2 a 2 c-a}, C L B_{2 a 2 c-m}, \\
C L B_{2 b}, C L B_{2 d-m}, \\
P_{R}, P_{D}, P_{S}, P_{Y} \\
\bar{x}_{1}^{1}, \sigma_{1}^{1}, \ldots, \bar{x}_{N}^{M^{\max }}, \sigma_{N}^{M^{\max }} \\
a_{0}^{1}, \ldots, a_{0}^{M^{\max }}, a_{1}^{1}, \ldots, a_{N}^{M^{\max }}
\end{array}\right\}
$$

where: $\left[r a^{1}, \ldots, r a^{M^{\max }}\right]$ - rule activation vector, $M^{\max }$ - maximum number of system rules, ICIndex and $O C I n d e x$ takes values from Tables 6 , and 7 respectively, $P_{R}, P_{D}, P_{S}, P_{Y}$ - are the parameters defined in the equations (17), (34), (18), (25) respec- tivelly, $\bar{x}_{1}^{1}, \sigma_{1}^{1}, \ldots, \bar{x}_{N}^{M^{\max }}, \sigma_{N}^{M^{\max }}$ - are the membership function parameters, $N$ - number of the system inputs, $a_{0}^{1}, \ldots, a_{0}^{M^{\max }}, a_{1}^{1}, \ldots, a_{N}^{M^{\max }}$ - are the function parameters for the Takagi-Sugeno part of the system.

The number of the rules used to determine each of the evaluation criteria shown in Table 8 is determined by the formula

$$
M=\sum_{j=1}^{M^{\max } r a^{j},}
$$

where: $\left[r a^{1}, . ., r a^{M^{\max }}\right]$ contains the binary values $\{0,1\}$ obtained according to the formula (60).

\section{The problem of the function ap- proximation}

In this section, we will show the effectiveness of the proposed hardware implementation of the HRBF-TS system using the hybrid population algorithm (FSHPSO-AA). This will be demonstrated on a real-world problem modeling a nonlinear relationship $L_{q}\left(i_{q}, \Theta\right)$, the form of which was taken from the publication [3]. The exact numerical form of the modeled relationship was not known to the authors of this publication, either in the form of lookup tables or in analytical form. Thus, to carry out the experiment involving the modeling of the relationship $L_{q}\left(i_{q}, \Theta\right)$, the following function of two variables was selected according to the monograph [3].

$$
\begin{aligned}
y & =0.047-0.01 x_{1} \\
& -\left(0.001+0.0015 x_{1}\right) \cdot \cos \left(x_{2} 6 \pi\right)
\end{aligned}
$$

where $x_{1}, x_{2} \in[0,1]$.

Keep in mind that the task of designing the structure of the hardware implementation of the HRBF-TS system is to reproduce as accurately as possible the shape of a function of two variables, thus the lack of full correspondence of this function with the actual physical object of the device is not important in this case. 


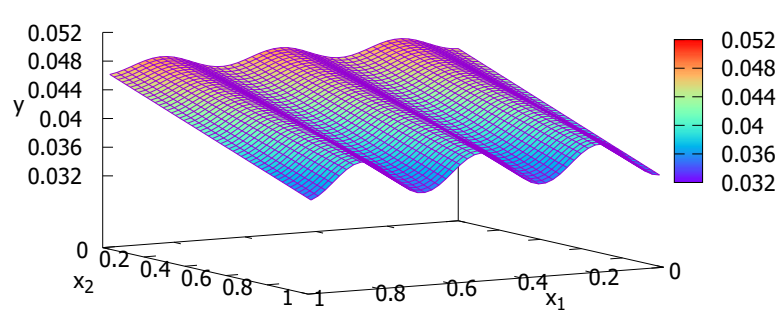

Figure 15. Approximated function according to the equation (64).

The approximated function of two variables described by the formula (64) is characterized by cyclicity, which results from the specificity of the modeled electromechanical system. In the considered modeling, it is sufficient to reproduce only one such cycle and to use it also for the other two cycles.

For the problem defined in this way, the following ranges were defined for the input variables: $x_{1} \in[-0.1,0.43]$ (1 period), $x_{2} \in[-0.1,1.1]$ and the output $y \in[0.032,0.052]$. Moreover, the maximum number of rules was set to be 9 , which results from dividing the 2-dimensional input data space into the three compartments for each input.Assuming fuzzy sets are no shared between the fuzzy rules, is needed 9 fuzzy sets per input - a total of 18 fuzzy sets for all rules (or 9 two-dimensional HRBF fuzzy sets).

For the above assumptions, the limit values from the Table 8 , and the proposed modification of the quality evaluation of the hardware implementation (56), the FSHPSO-E-AA algorithm was used. The task of the algorithm relies on estimating parameters, the number of rules, and the coding used for the implementation of the MRBF-TS system on the FPGA hardware platform. The parameters were coded according to the equation (62).

\section{Simulations and results}

The simulation tests of the hardware implementation of the MRBF-TS system were carried out using the implemented emulator of arithmetic operations with different precision. The task of the emulator is to emulate the performance of fixed-point/floating-point arithmetic operations with varying precision at various stages of calcula- tions. Due to the possibility of large values appearing at the calculation stages $(1 \mathrm{a}, 2 \mathrm{a}, 2 \mathrm{c})$ - for example, $s^{j}$ values determined using the equation (4), it was necessary to properly plan the evaluation of the obtained hardware implementation of the MRBFTS system.

So, the evaluation of the hardware implementation was done in two steps. Step 1 performs the maximum number of bits allocated to the integer part of the real number at particular stages of the calculations. It is realized through a full approximation of the shape of the function. In the second step, the precision of the system operation is evaluated, taking into account the fixed decimal point defined in step 1. To sum up - to evaluate a single solution, a two-fold approximation of the function course is necessary.

Table 9. The obtain parametrs of the MRBF-TS system (only active rules)

\begin{tabular}{c|c|c} 
& Takagi-Sugeno & Membership functions \\
\hline \hline $\mathrm{j}$ & $a_{0}^{j}, a_{1}^{j}, a_{2}^{j}$ & $\bar{x}_{1}^{j}, \sigma_{1}^{j}, \bar{x}_{2}^{j}, \sigma_{2}^{j}$ \\
\hline 1 & $0.56,-0.16,1.39$ & $0.25,1.11,-1.04,1.23$ \\
\hline 2 & $0.37,-0.015,-1.09$ & $0.22,1.29,-1.01,1.23$ \\
\hline 6 & $-0.57,-1.23,-1.68$ & $0.25,0.96,0.55,0.69$ \\
\hline 7 & $0.47,-1.05,-0.101$ & $0.49,1.15,0.43,1.54$ \\
\hline 9 & $-1.58,-1.96,0.137$ & $0.04,1.38,-0.63,0.49$
\end{tabular}

The simulation tests were carried out for the algorithm settings developed in the work [16], for the 50 particles. The maximum number of evaluations of the obtained solution was set to 50000. The evaluation of the hardware implementation of the HRBF-TS system was performed using the quality evaluation described by the formula (56) taking into account all the previously described criteria. As a result of using the algorithm, the hardware implementation of the MRBF-TS system for the function approximation (64) is obtained and presented in the Figure 16b. The obtained error is shown in Figure 16c. The progress of the FSHPSO-E-AA hybrid algorithm with all the components of the evaluation criteria is shown in Figure 17. 
b) The approximated function

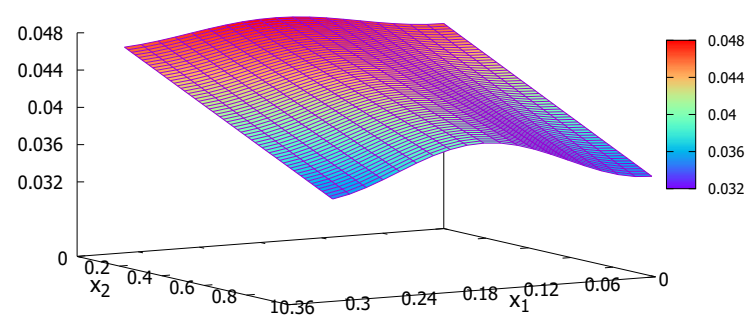

b) The obtained approximation using MRBF-TS

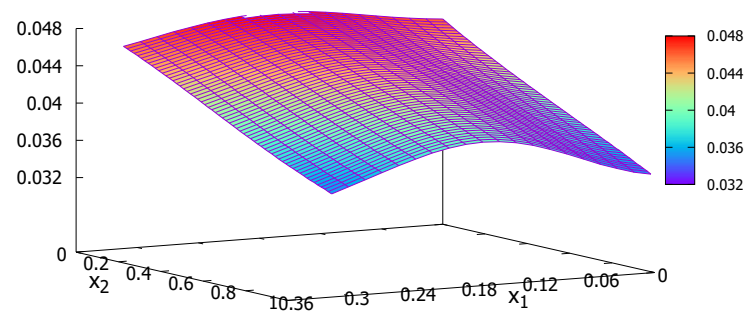

c) Error of the approximation

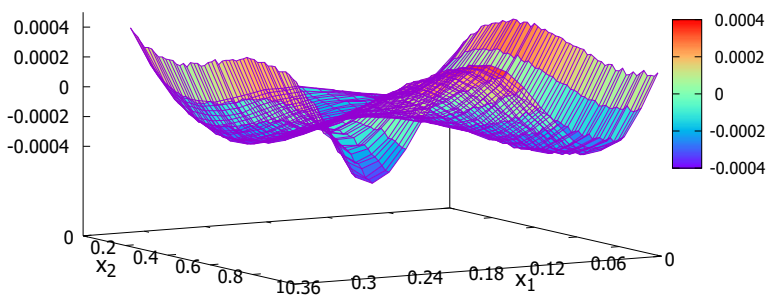

Figure 16. The results of the experiment: (a) - the approximated function, (b) - the obtained approximation using the hardware implementation of the MRBF-TS system, (c) - the obtained error of the approximation $\left(y-y^{*}\right)$

a) Accuracy of the hardware implementation

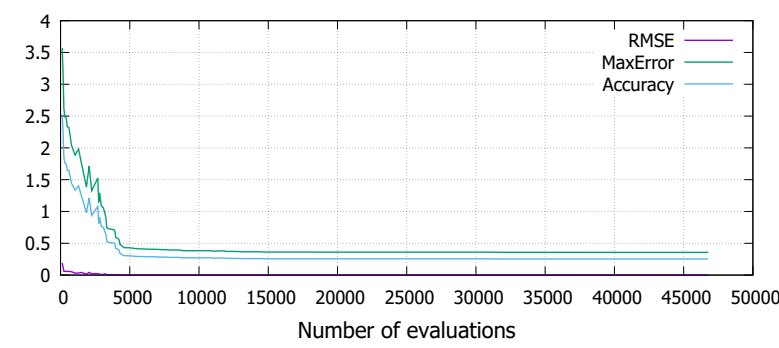

b) Cost of the hardware implementation

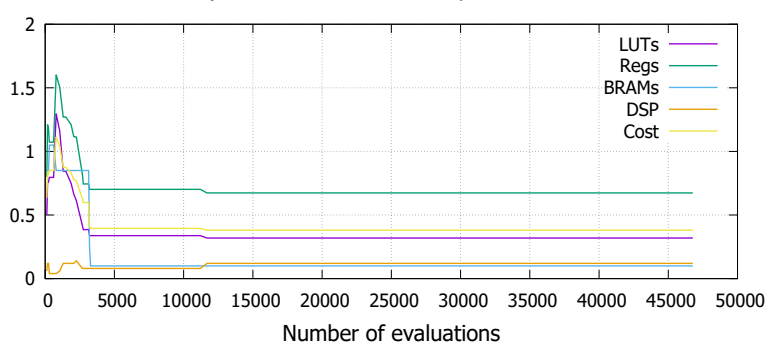

c) Quality of the hardware implementation

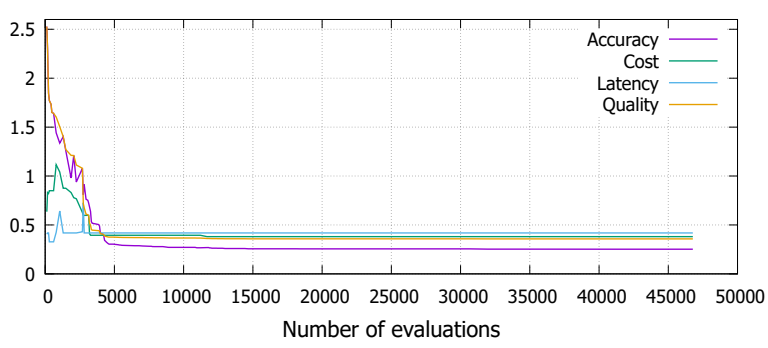

Figure 17. The progress of the FSHPSO-E-AA algorithm - changes of the accuracy (a), cost (b) and quality (c) evaluation in the function of evaluations number.

The parameters of the obtained hardware implementation of the HRBF-TS system are shown in Tables 9 , and 10 .

As can be observed in Table 10, the population algorithm chose the FX12 coding to perform the calculation in stages $1 \mathrm{a}$ and $1 \mathrm{~b}$. On the other hand, stages 1c, 2a, 2c, and $2 \mathrm{~b}$ were performed with the precision of FX14 coding. Stage $2 b$ is calculated with the precision FX16 and is responsible for calculating the reciprocal function - is realized with the greatest precision. Moreover, the hardware implementation of the MRBF-TS system obtains $R M S E=0.0015 \%$ and $\max \left|y-y^{*}\right|=0.71 \%$ which is consistent with the requirements defined in the Table 8. Also, the algorithm determined the implementation of arithmetic operations using CLB slides in steps 1a, 2b, and 2d.

The computations are performed in parallel according to the set values of the $P_{R}, P_{D}, P_{Y}$ parameters, but rules must be performed sequentially $P_{S}=$ 1. As a result of the analysis of the hardware implementation, the maximum values of the decimal point were established at individual stages of the calculations, which can be seen in Table 11. The largest value of the decimal point is determined in step 1a (3), which means that for small values, the numbers are written using only 9-bits. 
Table 10. The obtain parametrs of the hardware implementation

\begin{tabular}{l|c|l|c} 
Name & Value & Name & Value \\
\hline \hline ICIndex & 9 & $C L B_{2 a 2 c-m}$ & 0 \\
\hline OCIndex & 7 & $C L B_{2 b}$ & 1 \\
\hline$C L B_{1 a-a}$ & 1 & $C L B_{2 d-m}$ & 1 \\
\hline$C L B_{1 a-m}$ & 1 & $P_{R}$ & 1 \\
\hline$C L B_{1 c-a}$ & 0 & $P_{D}$ & 1 \\
\hline$C L B_{1 c-m}$ & 0 & $P_{S}$ & 1 \\
\hline$C L B_{2 a 2 c-a}$ & 0 & $P_{Y}$ & 1 \\
\hline
\end{tabular}

Table 11. The obtained decimal positions (DP) on each stage of the calculations.

\begin{tabular}{l|c|l|c} 
Stage & DP & Stage & DP \\
\hline \hline $1 \mathrm{a}$ & 3 & $1 \mathrm{c}$ & 2 \\
\hline $2 \mathrm{a}$ & 2 & $2 \mathrm{~b}$ & 0 \\
\hline $2 \mathrm{c}$ & 2 & $2 \mathrm{~d}$ & 2 \\
\hline
\end{tabular}

\section{Conclusions}

This study proposes a new method for the hardware implementation of the Takagi-Sugeno neurofuzzy system using the FSHPSO-E-AA hybrid population algorithm. The proposed method demonstrates high efficiency in determining all parameters of the hardware implementation of the MRBFTS system, which confirms usefulness developed earlier FSHPSO-E-AA hybrid algorithm developed and presented in the work [16].

In the proposed hardware implementation method, the cost of the implementation of individual arithmetic operations was determined for different method of encoding real numbers on FPGAs. The costs take into account the kind of resources used, i.e. DSP slice and LUTs. For some functions, like exponential and reciprocal, the LUT based method was also considered. A dedicated method of aggregating the evaluation criteria of the hardware implementation was developed, which enables the expert to define the requirements in the form of permissible limit values.

The developed hardware implementation method was tested on a practical example of approximating the shape of a function describing the behavior of a physical object. As a result, the hardware implementation of the MRBF-TS system that meets all defined criteria (limit values) was ob- tained. The proposed new quality evaluation function for the hardware implementation of the MRBFTS system turned out to be a very effective solution, as evidenced by the obtained simulation results.

\section{Acknowledgement}

The project was financed under the program of the Polish Minister of Science and Higher Education under the name "Regional Initiative of Excellence" in the years 2019 - 2022 project number $020 / \mathrm{RID} / 2018 / 19$, the amount of financing $12,000,000.00$ PLN.

\section{References}

[1] J. R. Jang and C. T. Sun, "Functional equivalence between radial basis function networks and fuzzy inference systems," IEEE Trans Neural Netw, vol. 4, no. 1, pp. 156-159, 1993.

[2] A. Przybył and M. J. Er, "The method of hardware implementation of fuzzy systems on FPGA," in Artificial Intelligence and Soft Computing (L. Rutkowski, M. Korytkowski, R. Scherer, R. Tadeusiewicz, L. A. Zadeh, and J. M. Zurada, eds.), (Cham), pp. 284-298, Springer International Publishing, 2016.

[3] A. Przybył, Algorytmy inteligencji obliczeniowej dla rozproszonych środowisk sieciowych. EXIT, 2017.

[4] A. Przybył and M. J. Er, "A method for design of hardware emulators for a distributed network environment," in Artificial Intelligence and Soft Computing (L. Rutkowski, M. Korytkowski, R. Scherer, R. Tadeusiewicz, L. A. Zadeh, and J. M. Zurada, eds.), (Cham), pp. 318-336, Springer International Publishing, 2017.

[5] J. Detrey and F. de Dinechin, "Parameterized floating-point logarithm and exponential functions for FPGAs," Microprocessors and Microsystems, vol. 31, no. 8, pp. 537-545, 2007. Special Issue on FPGA-based Reconfigurable Computing (3).

[6] P. Echeverria and M. Lopez-Vallejo, "An FPGA implementation of the powering function with single precision floating-point arithmetic," in High Performance Digital Design in Reconfigurable Architectures, pp. 17-26, 8th Conference on Real Numbers and Computers, 2008.

[7] J. Kluska and Z. Hajduk, "Hardware implementation of P1-TS fuzzy rule-based systems on FPGA," in Artificial Intelligence and Soft Computing, 12th 
International Conference, ICAISC, Part I, vol. 7894, pp. 282-293, 2013.

[8] J.-Y. Jhang, K.-H. Tang, C.-K. Huang, C.-J. Lin, and K.-Y. Young, "FPGA implementation of a functional neuro-fuzzy network for nonlinear system control," Electronics, vol. 7, no. 8, 2018.

[9] M. Dendaluce Jahnke, F. Cosco, R. Novickis, J. Pérez Rastelli, and V. Gomez-Garay, "Efficient neural network implementations on parallel embedded platforms applied to real-time torque-vectoring optimization using predictions for multi-motor electric vehicles," Electronics, vol. 8, no. 2, 2019.

[10] A. Brown, P. Kelly, and W. Luk, "Profiling floating point value ranges for reconfigurable implementation," 012007.

[11] A. Agrawal, J. Choi, K. Gopalakrishnan, S. Gupta, R. Nair, J. Oh, D. A. Prener, S. Shukla, V. Srinivasan, and Z. Sura, "Approximate computing: Challenges and opportunities," in 2016 IEEE International Conference on Rebooting Computing (ICRC), pp. 1-8, 2016.

[12] D. Han, S. Zhou, T. Zhi, Y. Wang, and S. Liu, "Float-fix: An efficient and hardware-friendly data type for deep neural network," International Journal of Parallel Programming, vol. 47, no. 3, pp. 345-359, 2019.

[13] A. Przybył and J. Szczypta, "Method of evolutionary designing of FPGA-based controllers," Przegląd Elektrotechniczny, vol. 92, no. 7, pp. 174-179, 2016.

[14] R. Eberhart and J. Kennedy, "A new optimizer using particle swarm theory," in MHS'95. Proceedings of the Sixth International Symposium on Micro Machine and Human Science, pp. 39-43, IEEE, 1995.

[15] P. Dziwiński and Ł. Bartczuk, "A new hybrid particle swarm optimization and genetic algorithm method controlled by fuzzy logic," IEEE Transactions on Fuzzy Systems, vol. 28, no. 6, pp. 1140-1154, 2019.

[16] P. Dziwiński, Ł. Bartczuk, and J. Paszkowski, “A new auto adaptive fuzzy hybrid particle swarm optimization and genetic algorithm," Journal of Artificial Intelligence and Soft Computing Research, vol. 10, pp. 95-111, 2020.

[17] K. Łapa, K. Cpałka, Ł. Laskowski, A. Cader, and Z. Zeng, "Evolutionary algorithm with a configurable search mechanism," Journal of Artificial Intelligence and Soft Computing Research, vol. 10, pp. 151-171, 2020 .

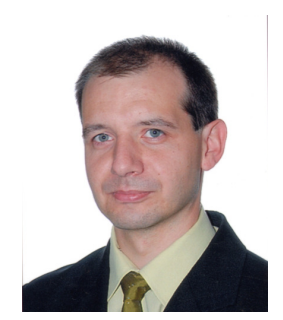

Piotr Dziwiński was born in Zawiercie, Poland, in 1978. He received the MSc degree in 2003 and the $\mathrm{PhD}$ degree in 2008 in computer science, both from the Czestochowa University of Technology. Since 2008 he has been an Assistant Professor at the Institute of Computational Intelligence, Czestochowa University of Technology, Czestochowa, Poland. He has published about 20 technical papers in conference proceedings and book chapters. His main fields of interests include swarm intelligence, evolutionary algorithms, hybrid methods and fuzzy systems.

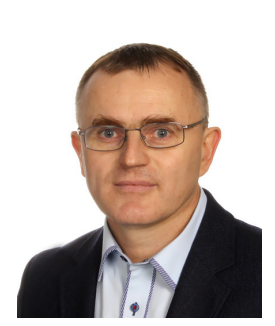

Andrzej Przybyl is an Associate Professor at the Department of Intelligent Computer Systems, Czestochowa University of Technology, Poland. His research interests center on designing intelligent control systems, including hardware implementation of soft computing algorithms on field-programmable gate arrays (FPGAs). He is working on the development of new control algorithms of computer numerically controlled (CNC) machines, real-time Ethernet communication solutions, and hardware-in-the-loop (HIL) systems. He has published over 40 technical papers.

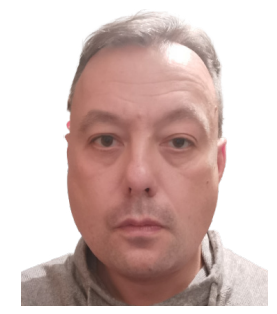

Pawel Trippner is a professor at University of Social Sciences in Łódź, Poland. He received the Ph.D. degree in economic sciences if the field of economics from the University of Łódź, Poland. His research interest includes the issues of corporate finance management, capital market investments and applications of artificial intelligence methods in management.

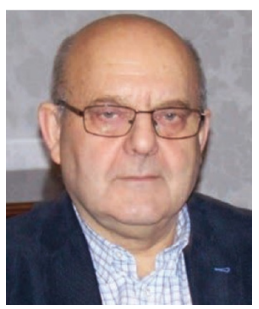

Józef Paszkowski graduated from the Military University of Technology i Warsaw, Faculty of Cybernetics (M.Sc. 1976) with specialization in organization and design of IT systems. He completed post-graduate studies in management systems at the University of Lodz and programming technology at the University of Warsaw/IMM PAN in Warsaw. In 1995 he received the Ph.D. in the field of medical informatics. In the years 2000-2004 he worked as specialist in a IT company. Currently, he is an Assistant Professor at the University of Social Science in Łódź. He specializes in analyzing and modeling data processing system for distributed business and public utilities. His research and teaching interest include database systems, process management methods, information systems design technologies, computational intelligence, and knowledge engineering methods. 


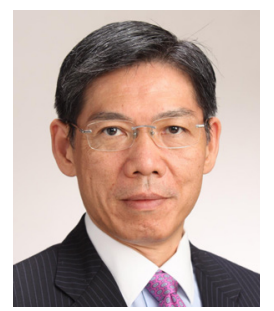

Prof. Yoichi Hayashi received the Dr. Eng. degree in systems engineering from Tokyo University of Science, Tokyo in 1984. In 1986, he joined the Computer Science Department of Ibaraki University, Japan, as an Assistant Professor. Since 1996, he has been a Full Professor at Computer Science Department, Meiji University, Tokyo. He was a visiting professor at the University of Alabama at Birmingham and University of Canterbury (New Zealand). He authored over 230 published computer science papers. His current research interests include explainable AI, deep learning, rule extraction, high-performance classifiers, and medical informatics and medical imaging. He has been the Action Editor of Neural Networks and the Associate Editor of IEEE Trans. Fuzzy Systems. He has served as Editor-in-Chief and Associate Editor, Editorial Board Member, Review Board Member, Guest Editor and Reviewer in 60 academic journals, and was involved in the work for the European Research Council Executive Agency, National Sciences and Engineering Research Council of Canada (NSERC). He has been a senior member of the IEEE since 2000. 